\title{
3D Modelling of the climatic impact of outflow channel formation events on Early Mars.
}

\author{
Martin Turbet ${ }^{1}$, Francois Forget ${ }^{1}$, James W. Head ${ }^{2}$, and Robin \\ Wordsworth $^{3}$ \\ ${ }^{1}$ Laboratoire de Météorologie Dynamique, Sorbonne Universités, \\ UPMC Univ Paris 06, CNRS, 4 place Jussieu, 75005 Paris. \\ ${ }^{2}$ Department of Earth, Environmental and Planetary Sciences, \\ Brown University, Providence, RI 02912, USA. \\ ${ }^{3}$ Paulson School of Engineering and Applied Sciences, Harvard \\ University, Cambridge, MA 02138, USA.
}

June 29, 2021 


\begin{abstract}
Mars was characterized by cataclysmic groundwater-sourced surface flooding that formed large outflow channels and that may have altered the climate for extensive periods during the Hesperian era. In particular, it has been speculated that such events could have induced significant rainfall and caused the formation of late-stage valley networks. We present the results of 3-D Global Climate Model simulations reproducing the short and long term climatic impact of a wide range of outflow channel formation events under cold ancient Mars conditions. We find that the most intense of these events (volumes of water up to $10^{7} \mathrm{~km}^{3}$ and released at temperatures up to 320 Kelvins) cannot trigger long-term greenhouse global warming, regardless of how favorable are the external conditions (e.g. obliquity and seasons). Furthermore, the intensity of the response of the events is significantly affected by the atmospheric pressure, a parameter not well constrained for the Hesperian era. Thin atmospheres $(\mathrm{P}<80$ mbar) can be heated efficiently because of their low volumetric heat capacity, triggering the formation of a convective plume that is very efficient in transporting water vapor and ice at the global scale. Thick atmospheres $(\mathrm{P}>0.5$ bar) have difficulty in producing precipitation far from the water flow area, and are more efficient in generating snowmelt. In any case, outflow channel formation events at any atmospheric pressure are unable to produce rainfall or significant snowmelt at latitudes below $40^{\circ} \mathrm{N}$. As an example, for an outflow channel event (under a 0.2 bar atmospheric pressure and $45^{\circ}$ obliquity) releasing $10^{6} \mathrm{~km}^{3}$ of water heated at 300 Kelvins and at a discharge rate of $10^{9} \mathrm{~m}^{3} \mathrm{~s}^{-1}$, the flow of water reaches the lowest point of the northern lowlands (around $\sim 70^{\circ} \mathrm{N}, 30^{\circ} \mathrm{W}$ ) after $\sim 3$ days and forms a $200 \mathrm{~m}$-deep lake of $4.2 \times 10^{6} \mathrm{~km}^{2}$ after $\sim 20$ days; the lake becomes entirely covered by an ice layer after $\sim 500$ days. Over the short term, such an event leaves $6.5 \times 10^{3} \mathrm{~km}^{3}$ of ice deposits by precipitation ( $0.65 \%$ of the initial outflow volume) and can be responsible for the melting of $\sim 80 \mathrm{~km}^{3}$ ( $0.008 \%$ of the initial outflow volume; $1 \%$ of the deposited precipitation). Furthermore, these quantities decrease drastically (faster than linearly) for lower volumes of released water. Over the long term, we find that the presence of the ice-covered lake has a climatic impact similar to a simple body of water ice located in the Northern Plains.

For an obliquity of $\sim 45^{\circ}$ and atmospheric pressures $>80$ mbar, we find that the lake ice is transported progressively southward through the mechanisms of sublimation and adiabatic cooling. At the same time, and as long as the initial
\end{abstract}


water reservoir is not entirely sublimated (a lifetime of $10^{5}$ martian years for the outflow channel event described above), ice deposits remain in the West Echus Chasma Plateau region where hints of hydrological activity contemporaneous with outflow channel formation events have been observed. However, because the high albedo of ice drives Mars to even colder temperatures, snowmelt produced by seasonal solar forcing is difficult to attain. 


\section{Introduction}

During the Late Hesperian epoch of the history of Mars (about 3.1-3.6 Gyrs ago; Hartmann and Neukum (2001)), the large outflow channels observed in the Chryse Planitia area are thought to have been carved by huge water floods caused by catastrophic and sudden release of groundwater (Baker, 1982; Carr, 1996). It has been speculated that such events could have warmed the climate and possibly explain the contemporaneous formation of dendritic valley networks observed in the nearby Valles Marineris area and on the flanks of Alba Patera, Hecates Tholus, and Ceraunius Tholus, and that have been interpreted to be precipitation-induced (Gulick and Baker, 1989, 1990; Baker et al., 1991; Gulick et al., 1997; Gulick, 1998, 2001; Mangold et al., 2004; Quantin et al., 2005; Weitz et al., 2008; Santiago et al., 2012). Although the Late Hesperian epoch is thought on the basis of geology and mineralogy to have been cold (Head et al., 2004; Bibring et al., 2006; Ehlmann et al., 2011), the characteristics of these dendritic valleys suggest that the valleys were formed under persistent warm conditions (.e.g, Mangold et al. 2004). First, their high degree of branching is interpreted to indicate formation by precipitation. Second, their high drainage densities - evidence of their high level of maturity - and the presence of inner channels favor the presence of stable liquid water for geologically long periods of time (Craddock and Howard, 2002). Third, sedimentary morphologies observed in the region of Valles Marineris (Quantin et al. 2005) suggest a fluvial and lacustrine environment. Under this hypothesis, the warm liquid water floods that formed the outflow channels would inject water vapor into the atmosphere, a powerful greenhouse gas that could trigger a significant warming period possibly leading to long lasting pluvial activity (rainfall).

In this paper, we use a 3-Dimensional Global Climate Model (LMD GCM) to explore the global climatic impact of outflow channel water discharge events on a Late Hesperian Mars over a range of temperatures and atmospheric pressures. These bursts of warm liquid groundwater outflows onto the surface can trigger strong evaporation, possibly leading to global climate change. How warm and how wet was the atmosphere of Late Hesperian Mars after such major outflow channel events? The climatic effect of relatively small and cool groundwater discharges has been studied on a regional scale (Kite et al., 2011a) and localized precipitation is indicated. In this contribution, we investigate the climatic impact at a global scale of a wide range of possible outflow channel events, including the case of the most intense outflow events ever recorded on Mars (Carr, 1996). Our work focuses on both (1) the direct short-term climate change due to the initial strong evaporation of water vapor and (2) the long-term change of the water cycle 
due to the presence of liquid water and ice at non-stable locations.

When a warm liquid water flow reaches the surface, strong evaporation occurs and the total evaporation rate increases with the temperature and the surface area of the flow. In term of energy budget, a $300 \mathrm{~K}$ warm liquid water flow can potentially convert $\sim 5 \%$ of its total mass into water vapor before freezing starts. The injected water vapor will have a major role on the radiative budget of the planet. First, water vapor is a greenhouse gas that can absorb ground thermal infrared emission efficiently. Second, water vapor can condense to form clouds. In the process, large amounts of latent heat can be released in the atmosphere. The clouds can reflect the incoming solar flux as well as contribute to an additional greenhouse effect, depending on their height and the opacity of the background atmosphere, which depends on the total atmospheric pressure.

To study the global climatic effect of localized outflow channel events, 3D Global Climate Models are particularly relevant because they not only model the physical processes described above, but also the $3 \mathrm{D}$ dynamical processes that play a major role in climatic evolution. In particular, we show in this paper that $3 \mathrm{D}$ dynamical processes (horizontal advection, in particular) are key to understanding the relaxation timescale of the Late Hesperian martian atmosphere immediately following major outflow channel events.

\section{Background}

\subsection{Outflow channels}

\subsubsection{Description}

Outflow channels are long (up to $\sim 2000 \mathrm{~km}$ ) and wide (up to $\sim 100 \mathrm{~km}$ ) valleys that were sculpted by large-scale debris-laden water flows (Baker, 1982; Baker et al., 1992; Carr, 1996). The most prominent martian outflow channels are located in the circum-Chryse area and debouch in the Chryse Planitia itself (Carr, 1996; Ivanov and Head, 2001).

Several processes have been suggested to have caused such outburst floods (Kreslavsky and Head, 2002). It is likely that the water that was released during these events come from subsurface aquifers (Clifford, 1993; Clifford and Parker, 2001). In this scenario, the temperature of the extracted subsurface water is controlled by the geothermal gradient and thus would depend on its initial depth of origin. During the Late Hesperian, when outflow channel events largely occured, this gradient could have been locally higher (Baker, 2001), because the circum-Chryse 
area is close to the volcanically active Tharsis region (Cassanelli et al., 2015). Therefore, the discharged water could have reached the surface at a maximum temperature of tens of degrees above the freezing point (Kreslavsky and Head, 2002). We note that the run-away decomposition of $\mathrm{CO}_{2}$ clathrate hydrate (Milton, 1974; Baker et al., 1991; Hoffman, 2000; Komatsu et al., 2000), proposed as a possible mechanism for the origin of the outflow water, cannot produce water temperature greater than $10 \mathrm{~K}$ above the freezing point. To a first approximation, and from a climatic point of view, the only difference between these two processes of liquid water discharge is the temperature of the water. Thus, we considered in this paper various cases ranging from 280 Kelvins to 320 Kelvins (see section 6.1.1).

Whatever the physical process operating, large amounts of water released at very high rates are needed at the origin of the water flow in order to explain the erosion of the circum-Chryse outflow channels. The quantity of water estimated to erode all the Chryse basin channels is $\sim 6 \times 10^{6} \mathrm{~km}^{3}$ assuming $40 \%$ by volume of sediment (Carr, 1996) but could possibly be much more if one assume lower sediment loads (Kleinhans, 2005, Andrews-Hanna and Phillips, 2007), which is, for example the case on Earth ( $\sim 0.1 \%$ of sediment by volume).

The different estimates of outflow channel single-event volumes, discharge rates and durations lead to a wide range of results, but two endmember scenarios can be defined and explored. On the one hand, some researchers estimated that only a limited number of very intense (volume up to $3 \times 10^{6} \mathrm{~km}^{3}$, discharge rates up to $10^{9} \mathrm{~m}^{3} \mathrm{~s}^{-1}$ ) outflow channel formation events actually occured (Rotto and Tanaka, 1992; Baker et al., 1991; Komatsu and Baker, 1997).

On the other hand, more recently, other researchers argued that outflow channels were formed by numerous individual small events (Andrews-Hanna and Phillips, 2007; Harrison and Grimm, 2008). This latter work implies water volumes from hundreds to thousands of $\mathrm{km}^{3}$, discharge rates of $10^{6}-10^{7} \mathrm{~m}^{3} \mathrm{~s}^{-1}$ for individual events and a minimum period between successive single events of $\sim 20$ martian years. These endmember estimates differ by several orders of magnitude, but in this paper, we explored the full range.

\subsubsection{Fate of the outflow channel liquid water flow}

In this section, we provide a description of the possible fate, and calculations of the possible velocities, of the outflow channel water; these will serve as input for the description of the liquid water runoff under various conditions in the GCM simulations.

The ejected liquid water flows from the circum-Chryse area all inevitably 
debouch into the basin of Chryse. However, Chryse Planitia is not a closed basin and if the total amount of water released in a single event is high enough, the water will spill into the Northern Plains (Ivanov and Head, 2001), flowing down on slopes inclined at $\sim 0.03^{\circ}$ for more than $2000 \mathrm{~km}$. This is an important point because, as the wetted area of the flow increases, the total rate of evaporation rises. The fate of the outflow channel liquid water flow can be subdivided into two steps:

1. First, the ground-surface liquid water flows 'inside' the outflow channels. The Reynolds Number $R e$ of such flows is given by

$$
R e=\rho U_{c} R_{c} / \mu
$$

with $U_{c}$ the mean water flow velocity in the channel, $R_{c}$ the hydraulic radius (see below) of the channel, $\rho$ the density and $\mu$ the viscosity of the flow. For most of the outfow channel events, this number must have been orders of magnitude higher than 500 (Wilson et al. 2004), meaning that the released ground water flows were turbulent.

The most accurate way (Bathurst, 1993; Wilson et al., 2004) to calculate the mean velocity of such flows is to use the Darcy-Weisbach equation:

$$
U_{c}=\left(8 g R_{c} \sin \alpha / f_{c}\right)^{1 / 2}
$$

with $g=g_{\text {mars }}=3.71 \mathrm{~m} \mathrm{~s}^{-2}$ the gravity on Mars, $\alpha$ the slope angle of the channel and $f_{c}$ a dimensionless friction factor which mostly depends on the bed roughness $z_{c}$ and the water depth $h$ of the flow. This factor can be expressed as follows (Wilson et al., 2004):

$$
\left(8 / f_{c}\right)^{1 / 2}=a \log _{10}\left(R_{c} / z_{c}\right)+b
$$

with $a$ and $b$ two empirical coefficients, which are respectively equal to 5.657 and 6.6303 if the bed roughness $z_{c}\left(z_{c}=10^{-2} \mathrm{~m}\right.$ here) is fixed (Knudsen and Katz, 1958): This leads equation (2) to the following equation:

$$
U_{c}=\left(g R_{c} \sin \alpha\right)^{1 / 2}\left(a \log _{10}\left(R_{c} / z_{c}\right)+b\right) .
$$

The hydraulic radius $R_{c}$ is defined as the cross-sectional area of the channel divided by its wetted perimeter:

$$
R_{c} \sim\left(W_{c} h\right) /\left(W_{c}+2 h\right),
$$


with $W_{c}$ the channel width and $h$ the flow depth. Because outflow channels are wider than deep ( $W_{c} \sim 10-100 \mathrm{~km}$ wide but $h \leq 1 \mathrm{~km}$ deep), the hydraulic radius $R_{c}$ can be replaced by the depth of the water flow $h$.

To estimate the velocity of the flow according to its discharge rate $Q=U_{c} W_{c} h$, we solve equation (4) using the Lambert special function W defined by $x=W\left(x e^{x}\right)$. We obtain:

$$
h=\left(\frac{3 \ln 10}{2 a W_{c}(g \sin \alpha)^{\frac{1}{2}}} \frac{Q}{W\left(\frac{3 \times 10^{\frac{3 b}{2 a}} \ln 10}{2 a z_{c}^{\frac{3}{2}} W_{c}(g \sin \alpha)^{\frac{1}{2}}} Q\right)}\right)^{\frac{2}{3}}
$$

and

$$
U_{c}=\left(\frac{2 a(g \sin \alpha)^{\frac{1}{2}} W\left(\frac{3 \times 10^{\frac{3 b}{2 a}} \ln 10}{2 a z_{c}^{\frac{3}{2}} W_{c}(g \sin \alpha)^{\frac{1}{2}}} Q\right)}{3 \ln 10 W_{c}^{\frac{1}{2}}}\right)^{\frac{2}{3}} Q^{\frac{1}{3}} .
$$

The high concentrations of sediments in the flows (up to $40 \%$ of the volume) can increase the volumetric mass density $\rho$ (initially of $\rho_{\text {water }} \sim 1000 \mathrm{~kg} \mathrm{~m}^{-3}$ ) by a factor of 2 and the viscosity $\mu$ (initially of $\mu_{\text {water,300K }} \sim 8 \times 10^{-4} \mathrm{~Pa} \mathrm{~s}$ ) by a factor of 16 (Andrews-Hanna and Phillips, 2007), reducing by almost 10 the corresponding Reynolds Number. Nonetheless, since both the sediment load (from 0.1 to $40 \%$ ) and the dependence of the friction factor $f_{c}$ on the Reynolds Number $R e$, are poorly known (Andrews-Hanna and Phillips, 2007), their effects were not taken into account in the flow depth/velocity calculations.

2. As soon as the water flow leaves its channel and reaches Chryse Planitia, the width of the flow strongly increases (up to $2000 \mathrm{~km}$ ) and the slope angle decreases down to $0.03^{\circ}$. The mean flow velocity and height both decrease (Figure 11) whereas the wetted area increases significantly, leading to even more evaporation. The water will eventually end up in the main topographic depression of Vastitas Borealis (around $-30^{\circ} / 70^{\circ}$ in longitude/latitude) building up with time. If the volume of water released by the outflow channel event is higher than $\sim 2.6 \times 10^{6} \mathrm{~km}^{3}$, the water will spill from the North Polar basin to the Utopia Basin, filling it potentially up to $1.1 \times 10^{6} \mathrm{~km}^{3}$ (Ivanov and Head, 2001). If the volume of water exceeds $3.7 \times 10^{6} \mathrm{~km}^{3}$, the two basins become connected. They can be filled up to few tens of millions of $\mathrm{km}^{3}$.

Once the flow stops, some water will possibly remain in local topographic depressions such as impact craters or tectonic basins, thereby contributing to extended evaporation.

If the volume of water or the temperature of the flow are too low, the liquid water flow can potentially freeze before reaching the lowest points of the northern 

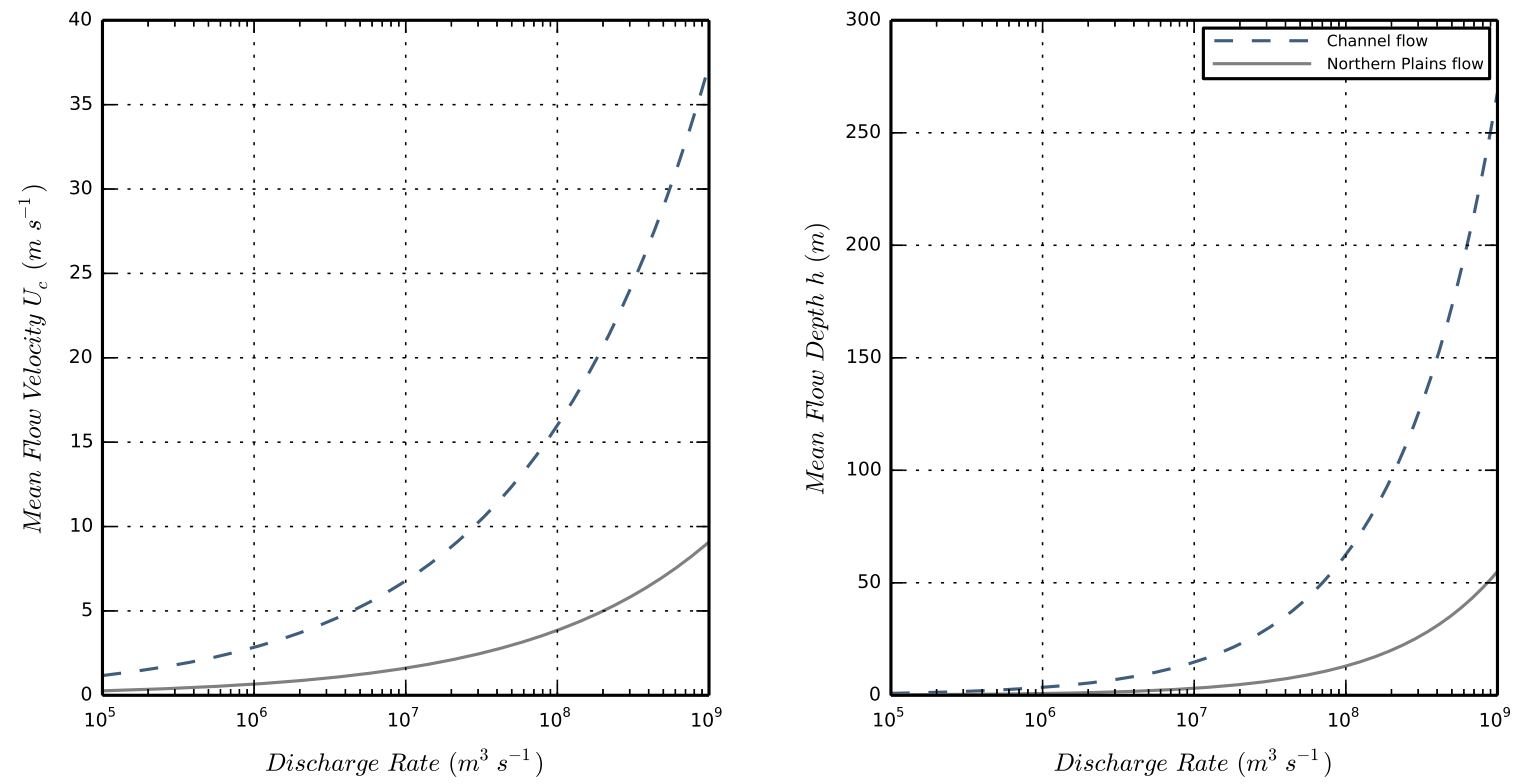

Figure 1: Estimates of mean flow velocity (left) and mean flow depth (right) for 1. (blue) the case of a $100 \mathrm{~km}$-wide channel flow on an $\sim 0.1^{\circ}$ slope angle and 2. (grey) the case for the same flow spilling onto the Northern Plains of Mars $\left(\sim 2000 \mathrm{~km}\right.$-wide and slope angle $\left.\sim 0.03^{\circ}\right)$. These quantities were calculated for a wide range of water discharge rates, using the Darcy-Weisbach equation. 
lowlands. This would likely occur only for the weakest outflow channel events (low volumes/discharge rates/temperatures), and we do not discuss this possibility further in this work.

\subsection{Late Hesperian Climate}

Late Hesperian Mars was likely to have been cold and dry globally, as suggested by the weak occurence of well-developed valley networks (Carr, 1996, Fassett and Head, 2008, Harrison and Grimm, 2005), the absence of observed phyllosilicates within layered deposits (Bibring et al., 2006; Chevrier et al., 2007; Carter et al., 2013), and the low erosion rates inferred from impact craters morphologies (Craddock and Maxwell, 1993; Golombek et al., 2006; Quantin et al., 2015).

As suggested by the stability of liquid water, and as supported by using the size distribution of ancient craters (Kite et al., 2014), the atmosphere of Mars at the end of the Noachian epoch was likely to have been thicker than the 8 mbar present day atmosphere. From the Noachian-Hesperian transition to the Late Hesperian era, magmatism may have been responsible for the build up of up to 400 mbar of $\mathrm{CO}_{2}$ in the atmosphere (Grott et al., 2011, Figure 4). In fact, it is during the period of formation of the outflow channels that the release of gaseous $\mathrm{CO}_{2}$ could have been at its maximum (Baker et al., 1991; Gulick et al., 1997): 1. Up to 100 mbars of $\mathrm{CO}_{2}$ could have been released by the contemporaneous Tharsis volcanism; 2. up to 60 mbars of $\mathrm{CO}_{2}$ per volume of $10^{6} \mathrm{~km}^{3}$ of outflow waters if produced by clathrate destabilization; and 3 . up to 20 mbars of $\mathrm{CO}_{2}$ per volume of $10^{6} \mathrm{~km}^{3}$ of outflow waters if coming from highly pressurized groundwater reservoirs saturated in $\mathrm{CO}_{2}$. However, most recent estimates of the several $\mathrm{CO}_{2}$ loss processes (photochemical escape, effect of solar wind, sputtering, impact erosion, loss to carbonates, etc.; summarized in Forget et al. (2013, section 3)) suggest that, in spite of the previously mentioned high estimates of $\mathrm{CO}_{2}$ outgassing amounts, it is very unlikely that the atmosphere of Late Hesperian Mars was thicker than 1 bar. In other words, there are currently no known physical/chemical processes that could accommodate the loss of an atmosphere at pressures of more than 1 bar.

To summarize, the Late Hesperian atmosphere was probably thicker than 8 mbar and thinner than 1 bar, but the actual surface pressure is still a matter of debate. In this paper, we find that the thickness of Late Hesperian Mars atmosphere plays an important role in relation to the climatic impact of outflow channel formation events. We chose to explore a wide possibility of atmospheric surface pressures, ranging from 40 mbar to 1 bar. 


\section{Model description}

\subsection{The Late Hesperian Global Climate Model}

In this paper we use the 3-Dimensions LMD Generic Global Climate Model, specifically developed for the study of the climate of ancient Mars (Forget et al., 2013; Wordsworth et al., 2013), and adapted here for the study of the influence of outflow channel events on Mars climate during the Late Hesperian.

This model is originally derived from the LMDz 3-dimensional Earth Global Climate Model (Hourdin et al., 2006), which solves the basic equations of geophysical fluid dynamics using a finite difference dynamical core on an Arakawa $\mathrm{C}$ grid.

The same model has been used to study many different planetary atmospheres including Archean Earth (Charnay et al., 2013), a highly irradiated 'future' Earth (Leconte et al., 2013a), Pluto (Forget et al., 2014), Saturn (Guerlet et al., 2014; Spiga et al., 2015) and exoplanets (Wordsworth et al., 2011, Leconte et al., 2013b; Forget and Leconte, 2014; Bolmont et al., 2016; Turbet et al., 2017, 2016).

Most of the simulations presented in this paper were performed at a spatial resolution of $96 \times 48\left(\right.$ e.g. $3.75^{\circ} \times 3.75^{\circ}$; at the equator, this gives in average $220 \mathrm{~km} \times 220 \mathrm{~km}$ ) in longitude / latitude. This corresponds approximately to twice the horizontal resolution used and eight times the calculation time needed in the work done by Forget et al. (2013) and Wordsworth et al. (2013). For this reason, a parallelized version of the GCM was used to deal with the long computation times. We explored the influence of the horizontal resolution (up to $1^{\circ} \times 1^{\circ} / 360 \times 180$ grid in longitude / latitude) and did not find any significant discrepency compared with the $96 \times 48$ lower resolution simulations.

In the vertical direction, the model is composed of 15 distinct atmospheric layers, generally covering altitudes from the surface to $\sim 50 \mathrm{~km}$. Hybrid $\sigma$ coordinates (where $\sigma$ is the ratio between pressure and surface pressure) and fixed pressure levels were used in the lower and the upper atmosphere, respectively. The lowest atmospheric mid-layers are located around [18, 40, 100, 230,..] meters and the highest at about [.., 20, 25, 35, 45] kilometers.

We used the present-day MOLA (Mars Orbiter Laser Altimeter) Mars surface topography (Smith et al., 1999; Smith et al., 2001), and we considered that most of the Tharsis volcanic load was largely in place by the end of the Hesperian epoch (Phillips et al. 2001).

We set the obliquity of Mars at $45^{\circ}$ to be consistent with both the most likely obliquity $\left(41.8^{\circ}\right)$ for ancient Mars calculated by Laskar et al. (2004) and one of the reference obliquities $\left(45^{\circ}\right)$ used in Wordsworth et al. (2013). The sensitivity 
of obliquity (and more generally of the seasonal effects) is discussed in section 4.

To account for the thermal conduction in the subsurface, we use an 18-layer thermal diffusion soil model that originally derives from Hourdin et al. 1993 and has been modified to take into account soil layers with various conductivities. The mid-layer depths range from $\mathrm{d}_{0} \sim 0.1 \mathrm{~mm}$ to $\mathrm{d}_{17} \sim 18 \mathrm{~m}$, following the power law $\mathrm{d}_{n}=\mathrm{d}_{0} \times 2^{n}$ with $n$ being the corresponding soil level, chosen to take into account both the diurnal and seasonal thermal waves. We assumed the thermal inertia of the Late Hesperian martian regolith to be constant over the entire planet and equal to $250 \mathrm{~J} \mathrm{~m}^{-2} \mathrm{~s}^{-1 / 2} \mathrm{~K}^{-1}$. This is slightly higher than the current Mars global mean thermal inertia in order to account for the higher atmospheric pressure.

Subgrid-scale dynamical processes (turbulent mixing and convection) were parameterized as in Forget et al.(2013) and Wordsworth et al. (2013). The planetary boundary layer was accounted for by the Mellor and Yamada (1982) and Galperin et al. (1988) time-dependent 2.5-level closure scheme, and complemented by a convective adjustment which rapidly mixes the atmosphere in the case of unstable temperature profiles (see section 3.1 .3 for more details).

In the simulations that include outflow channel events, the dynamical time step is $\sim 45$ seconds (respectively $\sim 184 \mathrm{~s}$ for the control simulations). The radiative transfer and the physical parameterizations are calculated every $\sim 15$ minutes and $\sim 4$ minutes (respectively every $\sim 1$ hour and $\sim 15$ minutes for the control simulations).

\subsubsection{Radiative Transfer in a $\mathrm{CO}_{2} / \mathrm{H}_{2} \mathrm{O}$ mixed atmosphere.}

The GCM includes a generalized radiative transfer for a variable gaseous atmospheric composition made of a mix of $\mathrm{CO}_{2}$ and $\mathrm{H}_{2} \mathrm{O}$ (HITRAN 2012 database, Rothman et al. (2013)) using the 'correlated-k' method (Fu and Liou, 1992)) suited for fast calculation. For this, we decomposed the atmospheric Temperatures / Pressures / Water Vapor Mixing Ratio into the following respective 7 × 8 × 8 grid: Temperatures $=\{100,150, . .350,400\} \mathrm{K}$; Pressures $=\left\{10^{-6}, 10^{-5}, . ., 1,10\right\}$ bar; $\mathrm{H}_{2} \mathrm{O}$ Mixing Ratio $=\left\{10^{-7}, 10^{-6}, . ., 10^{-2}, 10^{-1}, 1\right\} \mathrm{mol}$ of $\mathrm{H}_{2} \mathrm{O} / \mathrm{mol}$ of air $\left(\mathrm{H}_{2} \mathrm{O}+\mathrm{CO}_{2}\right.$ here $)$.

$\mathrm{CO}_{2}$ collision-induced absorptions (Gruszka and Borysow, 1998; Baranov et al., 2004: Wordsworth et al., 2010) ) were included in our calculations as in Wordsworth et al. (2013), as well as the $\mathrm{H}_{2} \mathrm{O}$ continuums. For this, we used the CKD model (Clough et al., 1989) with $\mathrm{H}_{2} \mathrm{O}$ lines truncated at $25 \mathrm{~cm}^{-1}$.

For the computation, we used 32 spectral bands in the thermal infrared and 35 in the visible domain. 16 non-regularly spaced grid points were used for the g-space integration, where $g$ is the cumulative distribution function of the 


\begin{tabular}{ll} 
Physical parameters & Values \\
& \\
\hline & \\
Mean Solar Flux & $465 \mathrm{~W} \mathrm{~m}^{-2}$ (79\% of present-day) \\
Obliquity & $45^{\circ}$ \\
Bare ground Albedo & 0.2 \\
Liquid water Albedo & 0.07 \\
$\mathrm{H}_{2} \mathrm{O}$ and $\mathrm{CO}_{2}$ ice Albedos & 0.5 \\
Surface Topography & Present-day \\
Surface Pressure & $0.2 \mathrm{bar}$ \\
Surface roughness coefficient & $0.01 \mathrm{~m}^{-2} \mathrm{~s}^{-1 / 2} \mathrm{~K}^{-1}$ \\
Ground thermal inertia & $250 \mathrm{~J} \mathrm{~m}^{-1}$
\end{tabular}

\section{Table 1: Physical Parameterization of the GCM.}

absorption data for each band. We used a two-stream scheme (Toon et al., 1989) to take into account the radiative effects of aerosols $\left(\mathrm{CO}_{2}\right.$ ice and $\mathrm{H}_{2} \mathrm{O}$ clouds $)$ and the Rayleigh scattering (mostly by $\mathrm{CO}_{2}$ molecules), using the method of Hansen and Travis (1974).

In summary, compared to the radiative transfer calculation used in Wordsworth et al. (2013), we utilized here a more recent spectroscopic database (HITRAN2012 instead of HITRAN2008) and built new correlated-k coefficients suited for wet atmospheres (water vapor VMR up to $100 \%$ ). In practice, the maximum water vapor Mass Mixing Ratio that was reached in our simulations (in the case of low surface pressure simulations) was $\sim 20 \%$.

In addition, we chose a mean solar flux of $465 \mathrm{~W} \cdot \mathrm{m}^{-2}$ (79\% of the present-day value of Mars; $35 \%$ of Earth's present-day value; and $105 \%$ of the flux used in the Wordsworth et al. (2013) work), corresponding to the reduced luminosity from standard solar evolution models (Gough, 1981) 3.0 Byrs ago, during the Late Hesperian era. During this epoch, the Sun was also $1.5 \%$ cooler (Bahcall et al., 2001); we did not, however, include in our model the resulting shift in the solar spectrum.

It is worth nothing anyway that absolute ages are based here on crater counting and are therefore not well constrained. For instance, the valley networks observed in West Echus Chasma Plateau are 2.9 to 3.4 billion years old (Mangold et al., 2004). 


\subsection{2 $\mathrm{CO}_{2}$ and Water cycles}

Both $\mathrm{CO}_{2}$ and $\mathrm{H}_{2} \mathrm{O}$ cycles are included in the GCM used in this work.

1. Carbon Dioxide is here the dominant gaseous species. In our model, $\mathrm{CO}_{2}$ can condense to form $\mathrm{CO}_{2}$ ice clouds and surface frost if the temperature drops below the saturation temperature. Atmospheric $\mathrm{CO}_{2}$ ice particles are sedimented and thus can accumulate at the surface. The $\mathrm{CO}_{2}$ ice layer formed at the surface can sublimate and recycle the $\mathrm{CO}_{2}$ in the atmosphere. The $\mathrm{CO}_{2}$ ice on the surface contributes to the surface albedo calculation: if the $\mathrm{CO}_{2}$ ice layer overpasses a threshold value of $1 \mathrm{~mm}$ thickness, then the local surface albedo is set immediately to the albedo of $\mathrm{CO}_{2}$ ice $(0.5$ in this work).

2. A self-consistent $\mathrm{H}_{2} \mathrm{O}$ water cycle is also included in the GCM. In the atmosphere, water vapor can condense into liquid water droplets or water ice particles, depending on the atmospheric temperature and pressure, forming clouds. At the surface, because the range of surface pressures modeled in this work are well above the triple point 6 mbar pressure, liquid water and water ice can coexist. Their contributions are both taken into account in the albedo calculation as in Wordsworth et al. (2013).

The stability of liquid water / ice / $\mathrm{CO}_{2}$ ice at the surface is governed by the balance between radiative and sensible heat fluxes (direct solar insolation, thermal radiation from the surface and the atmosphere, turbulent fluxes) and thermal conduction in the soil. Melting, freezing, condensation, evaporation, sublimation and precipitation physical processes are all included in the model.

\subsubsection{Convective Adjustment}

Outflow channel events result in the emplacement of warm liquid water, which leads to the sudden and intense warming of the atmosphere. Global Climate Models ( $200 \mathrm{~km}$ grid size for our simulations) are not suited to resolve the convection processes as is done in the case of mesoscale models, which have a typical km-size resolution (Kite et al., 2011a b).

Moist convection was taken into account following a moist convective adjustment that originally derives from the 'Manabe scheme' (Manabe and Wetherald, 1967; Wordsworth et al., 2013). In our scheme, relative humidity is let free and limited to $100 \%$, since it is inappropriate here to use an empirical value for relative humidity (versus altitude) that comes from Earth observations, as proposed in the original scheme.

This scheme has been chosen instead of more refined ones because it is: 1 . 
robust for a wide range of pressures; 2. energy-conservative; and 3. it is the most physically consistent scheme for exotic (non Earth-like) situations such as the ones induced by outflow channel events. In practice, when an atmospheric grid cell reaches $100 \%$ saturation and the corresponding atmospheric column has an unstable temperature vertical profile, the moist convective adjustment scheme is performed to get a stable moist-adiabatic lapse rate.

In our simulations, after major outflow channel events, large amounts of water vapor can be released into the atmosphere and the water vapor can easily become a dominant atmospheric species. In fact we recorded up to $20 \%$ water vapor Mass Mixing Ratios following intense outflow channels (in the case of low surface pressure). Thus, we used a generalized formulation of the moist-adiabiat lapse rate developed by Leconte et al. (2013a) (Supplementary Materials) to account for the fact that water vapor can become a main species in our simulations.

In our model we also used the numerical scheme proposed by Leconte et al. (2013a) to account for atmospheric mass change after the condensation or the evaporation of gases (water vapor in our case); this calculation is usually neglected in most of the well-known Global Climate Models. More details on the scheme can be found in Leconte et al. (2013a) (Supplementary Materials). This scheme comes from previous work for the $\mathrm{CO}_{2}$ cycle on present-day Mars (Forget et al. 1998), where there is some observational validation.

\subsubsection{Parameterization of the precipitation events}

$\mathrm{H}_{2} \mathrm{O}$ precipitation events were parameterized using a simple cloud water content threshold scheme (Emanuel and Ivkovi-Rothman, 1999) as in Wordsworth et al. (2013). If the cloud water content overpasses a threshold $l_{0}$ in a given atmospheric grid cell, precipitation occurs. We chose $1_{0}$ to be constant and equal to $0.001 \mathrm{~kg} / \mathrm{kg}$ as in Wordsworth et al. (2013). Wordsworth et al. (2013) examined the influence of $l_{0}$ and found it to be very low $\left(1 \mathrm{~K}\right.$ difference between $l_{0}=0.001$ and $0.01 \mathrm{~kg} / \mathrm{kg}$ ).

We note that the reevaporation of the precipitation is also taken into account in our numerical scheme.

\subsection{Control Simulations without outflow events}

We performed control simulations in the conditions described above for 5 different surface pressures (40 mbar, 80 mbar, 0.2 bar, 0.5 bar, 1 bar) and we obtained results which are consistent with Wordsworth et al. (2013) and Forget et al. (2013).

For these control runs, the three main differences between our work and Wordsworth 

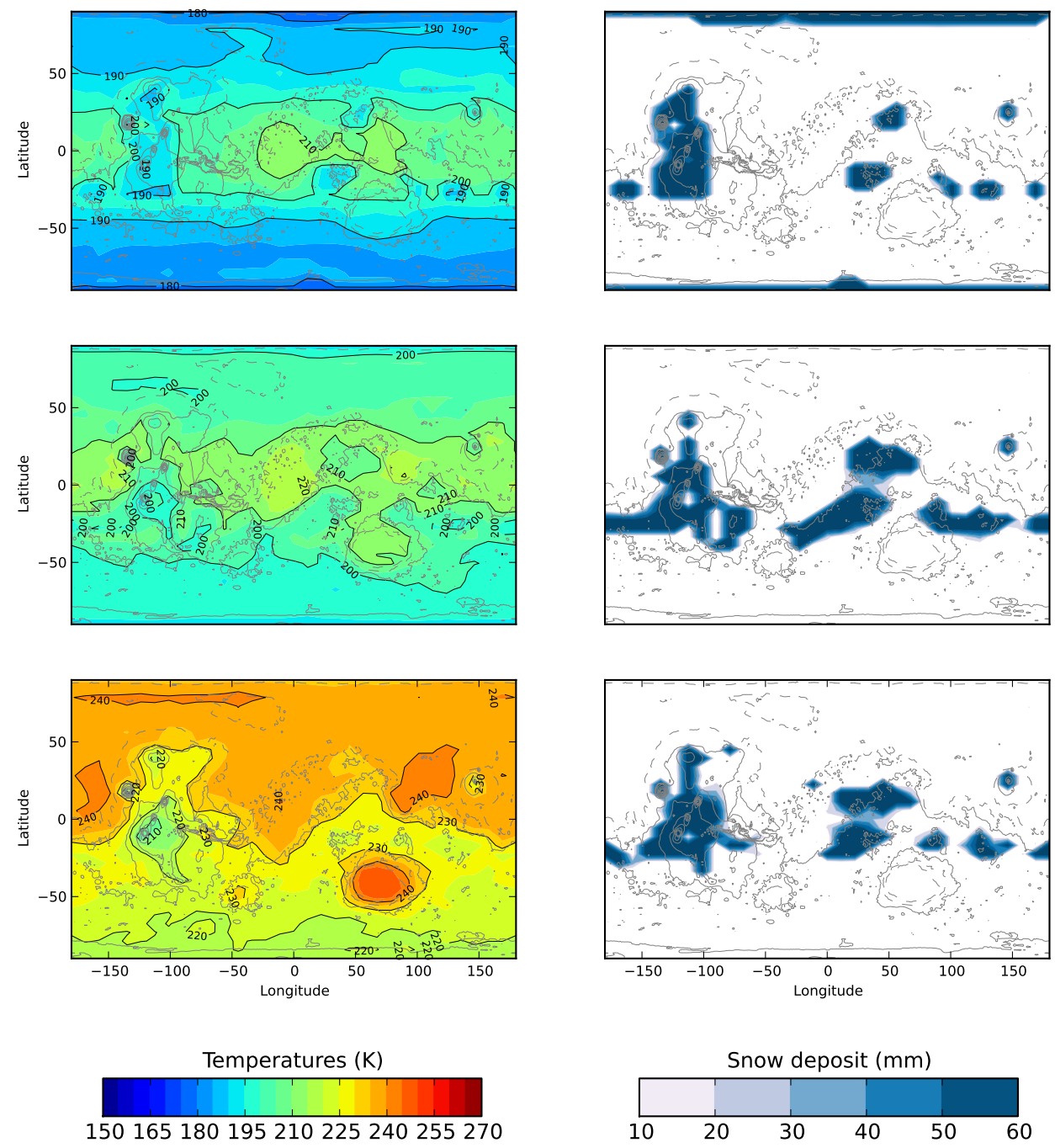

Figure 2: Surface Temperatures (left) and $\mathrm{H}_{2} \mathrm{O}$ ice deposit (right) annual means for the control simulations (at a $96 \times 48$ horizontal resolution) after $\sim 800$ martian years (or 30 loops of the ice iteration scheme). Top, middle and bottom plots correspond respectively to control simulations performed at 40 mbar, 0.2 bar and 1 bar. Grey contours show the topography used in the simulation. Ice iteration was performed every 2 years, with a 100-year timestep used for the first five iterations and 10-year timesteps used thereafter. 
et al. (2013) were: 1. the updated absorption coefficients (now HITRAN 2012); 2. an increase of the solar luminosity (now 79\% of Mars present-day value); and 3. the increase of the horizontal model resolution (from $32 \times 32$ to $96 \times 48$ in longitude $x$ latitude).

Figure 2 shows the mean annual surface temperatures and the position of the stable ice deposits for the reference case $(0.2$ bar) and the two surface pressure endmembers (40 mbar and 1 bar). The mean annual surface temperatures are slightly lower than in Figure 3 in Wordsworth et al. (2013) which were obtained for a fixed $100 \%$ relative humidity. It is also perhaps due to a slightly reduced $\mathrm{CO}_{2}$ ice cloud warming effect at high spatial resolution. The stable surface ice deposit locations were calculated using the ice equilibration algorithm of Wordsworth et al. (2013). Starting from a random initial surface ice distribution, (1) we run the GCM for two martian years then (2) we extrapolate the ice layer field $h_{\text {ice }}$ evolution calculation using:

$$
h_{\text {ice }}\left(t+n_{\text {years }}\right)=h_{\text {ice }}(t)+n_{\text {years }} \times \Delta h_{\text {ice }},
$$

with $\Delta h_{\text {ice }}$ the annual mean ice field change of the one-martian-year previous simulation and $n_{\text {years }}$ the number of years requested for the extrapolation. Then, (3) we eliminate of seasonal ice deposit and (4) we normalize the extrapolated ice field by the initial ice inventory to conserve the total ice mass. Eventually, (5) we repeat the process.

This algorithm has been shown (Wordsworth et al., 2013) to be insensitive to the proposed initial ice field location at the beginning of the simulation, at least assuming that the scheme has been repeated a sufficient number of times.

In total, for our control simulations, we performed the scheme 30 times, with $n_{\text {years }}=100$ for the first 5 loops and $n_{\text {years }}=10$ for 20 more loops for a resolution of $32 \times 32$. Then, we ran the algorithm 5 more times at the increased resolution of $96 \times 48$ to obtain a stable initial state necessary for the implementation of outflow channel events.

We note that 3D climate modeling under conditions similar to those described above (Forget et al., 2013; Wordsworth et al., 2013) have not yet been able to produce liquid water or at least significant precipitation by climatic processes anywhere on the planet, even when maximizing the greenhouse effect of $\mathrm{CO}_{2}$ ice clouds. 


\subsection{Experiment - Modeling of Outflow Channel Events}

\subsubsection{Description of the parameterization}

Outflow channel events can be modeled to a first approximation by the sudden release, and then the spread of warm liquid water over the surface of Mars. In our simulations, this was accomplished by the emplacement of a fully mixed layer of warm liquid water at the surface. The fate of this water depends on the following processes (summarized in Figure 3).:

1. The liquid water layer loses some energy by thermal conduction to the initially cold ground. For this, we fix the uppermost of the 18th martian regolith layers at the temperature of the water, and calculate the heat flux lost (or gained) by the warm water to the downward layers.

2. The warm liquid water layer cools by emitting thermal infrared radiation at $\sigma \mathrm{T}_{\text {surf }}^{4}$. This emission contributes to the radiative transfer budget.

3. The liquid water evaporates and looses some latent heat. The evaporation $E$ at the location of the warm water was computed within the boundary-layer scheme, using the following bulk aerodynamic formula:

$$
E=\rho_{1} C_{d} V_{1}\left[q_{\text {sat }}\left(T_{\text {surf }}\right)-q_{1}\right]
$$

where $\rho_{1}$ and $\mathrm{V}_{1}$ are the volumetric mass of air and the wind velocity at the first atmospheric level, $q_{\text {sat }}\left(T_{\text {surf }}\right)$ is the water vapor mass mixing ratio at saturation at the surface, and $q_{1}$ is the mixing ratio in the first atmospheric layer. The aerodynamic coefficient is given by $C_{d}=\left(\kappa / \ln \left(1+z_{1} / z_{0}\right)\right)^{2} \sim 2.5 \times 10^{-3}$, where $\kappa=0.4$ is the Von Karman constant, $z_{0}$ is the roughness coefficient and $z_{1}$ is the altitude of the first level ( $\sim 18$ meters).

We modeled the sensible heat exchanged between the surface and the first atmospheric layer using a similar formula:

$$
F_{\text {sensible }}=\rho_{1} C_{p} C_{d} V_{1}\left[T_{\text {surf }}-T_{1}\right]
$$

with $\mathrm{T}_{1}$ the temperature of the first atmospheric level and $\mathrm{C}_{p}$ the mass heat capacity assumed equal to $850 \mathrm{~J} \mathrm{~K}^{-1} \mathrm{~kg}^{-1}$ in case of a $\mathrm{CO}_{2}$-only atmospheric composition.

4. Depending on the volume of water modeled, liquid water will flow from the Circum-Chryse outflow channel sources to Chryse Planitia, then to Acidalia Planitia, and eventually to the Northern Plains. First, we modeled the displacement of the flow calculated from its height and its velocity. The velocity of the flow mostly depends on its width but also on the slope of the terrain. For each grid, we used the subgrid mean slope and the subgrid mean orientation of the slope to 
evaluate (using equations (4) and (7)) the velocity and the direction of the flow. Second, we used a simple bucket scheme to model the progressive filling of the topographic depressions.

Warm waters flowing on the Northern Plain slopes can also encounter $\mathrm{H}_{2} \mathrm{O}$ ice (it can be either stable at a particular latitude, or related to previous outflow channel events, but from the point of view of latent heat exchange and climate, it does not change anything) or seasonal $\mathrm{CO}_{2}$ ice (typically present for atmospheres thinner than 1 bar). We modeled the interaction of $\mathrm{H}_{2} \mathrm{O}$ and $\mathrm{CO}_{2}$ ices with warm liquid water using energy conservation. If the liquid water is warm and in a sufficient amount, all the $\mathrm{CO}_{2}$ ice sublimates and is added to the atmosphere. Similarly, all the water ice encountered by the warm flow is melted and converted at the resulting equilibrium temperature.

Once the flow has reached a stable position (e.g. forming a lake), in reality some water may be trapped in local topographic depressions (impact craters, tectonic basins, ...); it is difficult, however, to estimate adequately how much water might be sequestered in this manner. First, the detailed topography of the terrains is unknown prior to resurfacing by the outflow channel events. Second, the water outflows themselves modified (and probably smoothed) the topography. Thus, to take into account not only the effect of the trapped water but also the role of the wet ground, we arbitrarily placed a minimum $20 \mathrm{~cm}$ layer of liquid water in all the locations where the liquid water flow passed through. This assumption may also be representative of the fact that in reality the discharge rate does not have a rectangular shape (in time) as we assumed in our parameterizations.

5. As time goes on, the liquid water flow cools. If its temperature reaches the 273.15 K freezing temperature (assuming no salts), the water starts to freeze. On Earth, salinity drives the freezing point of oceans to $\sim 271 \mathrm{~K}$ and assuming similar salt rates in outflow waters would not change much our results. To account for this process, we developed a multiple layer modified version of the soil thermal conduction model already included in the GCM. We have in total 100+ layers, with mid-layer depths ranging from $\mathrm{d}_{0} \sim 0.1 \mathrm{~mm}$ to $\mathrm{d}_{14} \sim 2 \mathrm{~m}$, following the power law $\mathrm{d}_{n, n \leq 14}=\mathrm{d}_{0} \times 2^{n}$ with $n$ being the corresponding soil level and the linear law $\mathrm{d}_{n, n>14}=\mathrm{d}_{14} \times(n-13)$ for the deepest layers. The layers are separated into two parts: the ice cover above and the liquid water below. For the water ice layers, we use a thermal conductivity of $2.5 \mathrm{~W} \mathrm{~m}^{-1} \mathrm{~K}^{-1}$ and a volumetric heat capacity of $2 \times 10^{6} \mathrm{~J} \mathrm{~m}^{-3} \mathrm{~K}^{-1}$. For the liquid water, we use, respectively, a thermal inertia of $20000 \mathrm{~J} \mathrm{~m}^{-2} \mathrm{~K}^{-1} \mathrm{~s}^{-1 / 2}$ (artificially high to account for convection) and a volumetric heat capacity of $4 \times 10^{6} \mathrm{~J} \mathrm{~m}^{-3} \mathrm{~K}^{-1}$. At each physical timestep, we estimate the thermal diffusion flux lost by the liquid water layer to the water ice 
layer and calculate (using the conservation of energy) the amount of liquid water to freeze. If the depth of the ice - initially going down to $\mathrm{d}=\mathrm{d}_{n}$ - overpasses the layer $\mathrm{d}_{n+1}$, we convert the $n+1$ layer into ice.

We note that the use of a multi-layer soil model is important to describe the sea-ice formation, evolution and its impact on possible cold early martian climates. Such refined models are better suited to represent the temperature profile evolution within the ice layer (that may evolve with seasonal forcing or as the ice layer thickens) and thus the surface temperature that controls the sublimation rate. In particular, our simulations show that up to $95 \%$ of the annual sublimation rate can be produced during the summer seasons. This requires a good estimate of the seasonal variations of the surface temperature above the ice.

Simultaneously, as the ice layer forms, we also linearly increase the surface albedo from $\mathrm{A}_{\text {liq }}=0.07$ (if no ice) to $\mathrm{A}_{\text {ice }}=0.5$ (if the ice layer thickness $h$ overpasses the threshold value of $h_{*}=3.5 \mathrm{~cm}$; Le Treut and Li 1991) as follows:

$$
A=A_{\text {liq }}+\left(A_{\text {ice }}-A_{\text {liq }}\right) \frac{h}{h_{*}} .
$$

6. The amount of water delivered by outflow events can be very large and thus lead to the accumulation of large quantities of liquid water. The timing expected for this water to freeze can be evaluated using a combination of the thermal conduction flux in the ice layer $F=\lambda_{\text {ice }} \frac{\left(T_{\text {surf }}-T_{\text {bottom }}\right)}{h}$ and the conservation of energy. Assuming that the temperature in the frozen layer varies linearly between $T_{\text {bottom }}=273.15 \mathrm{~K}$ and $T_{\text {surf }}$ (assumed constant) as hypothesized in classical 2-layers thermodynamical models (Codron, 2012), we have:

$$
\rho_{\text {ice }}\left(L_{m}-C_{\text {ice }} \frac{\left(T_{\text {bottom }}-T_{\text {surf }}\right)}{2}\right) \frac{\partial h}{\partial t}=\lambda_{\text {ice }} \frac{\left(T_{\text {bottom }}-T_{\text {surf }}\right)}{h},
$$

where $\rho_{\text {ice }}$ is the volumetric mass of the ice $\left(9.2 \times 10^{2} \mathrm{~kg} \mathrm{~m}^{-3}\right), C_{\text {ice }}$ is the specific heat capacity of the ice $\left(2.1 \times 10^{3} \mathrm{~J} \mathrm{~kg}^{-1} \mathrm{~K}^{-1}\right), \lambda_{\text {ice }}$ is the conductivity of the ice $\left(2.5 \mathrm{~W} \mathrm{~m}^{-1} \mathrm{~K}^{-1}\right)$ and $L_{m} \sim 3.34 \times 10^{5} \mathrm{~J} \mathrm{~kg}^{-1}$ is the latent heat of fusion of water ice.

This leads after integration over time to an expression of $\mathrm{t}(\mathrm{h})$, the timing required to freeze a layer of depth $\mathrm{h}$ :

$$
t(h)=\frac{\rho_{\text {ice }}}{2 \lambda_{\text {ice }}}\left(\frac{L_{m}}{\left(T_{\text {bottom }}-T_{\text {surf }}\right)}-\frac{C_{\text {ice }}}{2}\right) h^{2} .
$$

For example, the outflow event presented in section 4 leads to the accumulation of up to 600 meters of liquid water. A typical timescale (for $T_{\text {surf }} \sim 200 \mathrm{~K}$ ) for this 


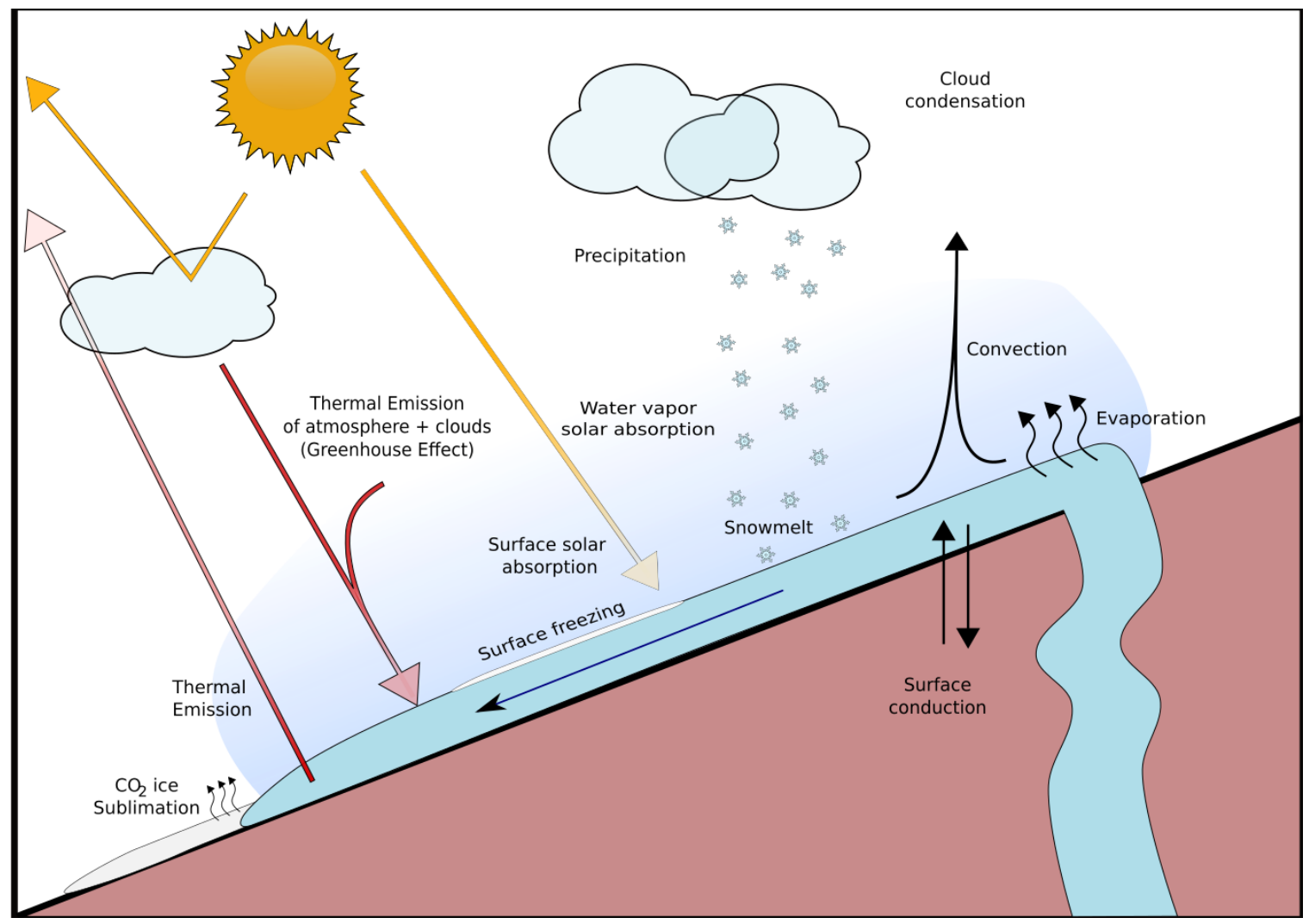

Figure 3: Schematic drawing of the physical processes taken into account during outflow channel events in our GCM simulations. 
water to freeze, according to equation 13 , is $\sim 4 \times 10^{3}$ martian years.

To account for such long timescales, we developed a modified version of the ice iteration scheme presented above. (1) First, we run the GCM for a few years then (2) every 2 years, we extrapolate the amount of ice that has locally condensed and sublimed in the simulations by an arbitrary factor $\mathrm{n}_{\text {years }}$. Simultaneously, (3) we proceed to a linear extrapolation of the amount of frozen water/of the growth of the ice layer thickness by the same factor $n_{\text {years }}$, using the conservation of energy. We actually fit the $t=f(h)$ function by straight lines of sizes multiple of $n_{\text {years }}$. In the reference simulation presented in section 4 , we performed first 5 martian years, then we extrapolated every 2 years using $\mathrm{n}_{\text {years }}=[5,5,20,20,50,50,100,100,500,500]$. After the extrapolation of the ice field/the ice layer depth is completed, (4) we arbitrarily set the ground temperature profile (where liquid water remains) to be linear, between $T_{\text {bottom }}=273.15 \mathrm{~K}$ and $T_{\text {surf }}$ calculated using the conservation of energy. This is a way to take into account (at first order) the evolution of the deepest ground layers that require very long timescales to stabilize their temperature profiles. The year following the extrapolation is thus also useful to get back a consistent temperature profile in the first layers (up to 15 meters typically).

7. Once the outflow water is completely frozen, we use again the ice iteration scheme (see section 3.2) to get estimates of the timing required for the ice to reach its stable positions.

\section{Results - the reference simulation}

We present in this section the results of simulations of outflow channel formation events occuring in the largest of the Circum Chryse channels: Kasei Vallis. We chose this particular location because 1. The Kasei Vallis outflow channel begins in Echus Chasma, which is close to the West Echus Chasma Plateau valley networks; and 2. Kasei Vallis is one of the largest outflow channels on Mars (Carr, 1996).

We focus first on a discharge of $10^{6} \mathrm{~km}^{3}$ (6.9 meters of GEL - Global Equivalent Layer) of liquid water heated at 300 Kelvins. Water is released at a constant rate of $10^{9} \mathrm{~m}^{3} \mathrm{~s}^{-1}$ in the region of Echus Chasma (see Figure 4 for the associated flow). This event is an upper estimate (in volume, discharge rate and temperatures) of the characteristics of outflow channel formation events (see section 2.1.1 for references).

As explained in section 2.2, surface atmospheric pressure in the Late Hesperian epoch is poorly constrained. Thus, we focus first on the case of a surface pressure of 0.2 bar. 

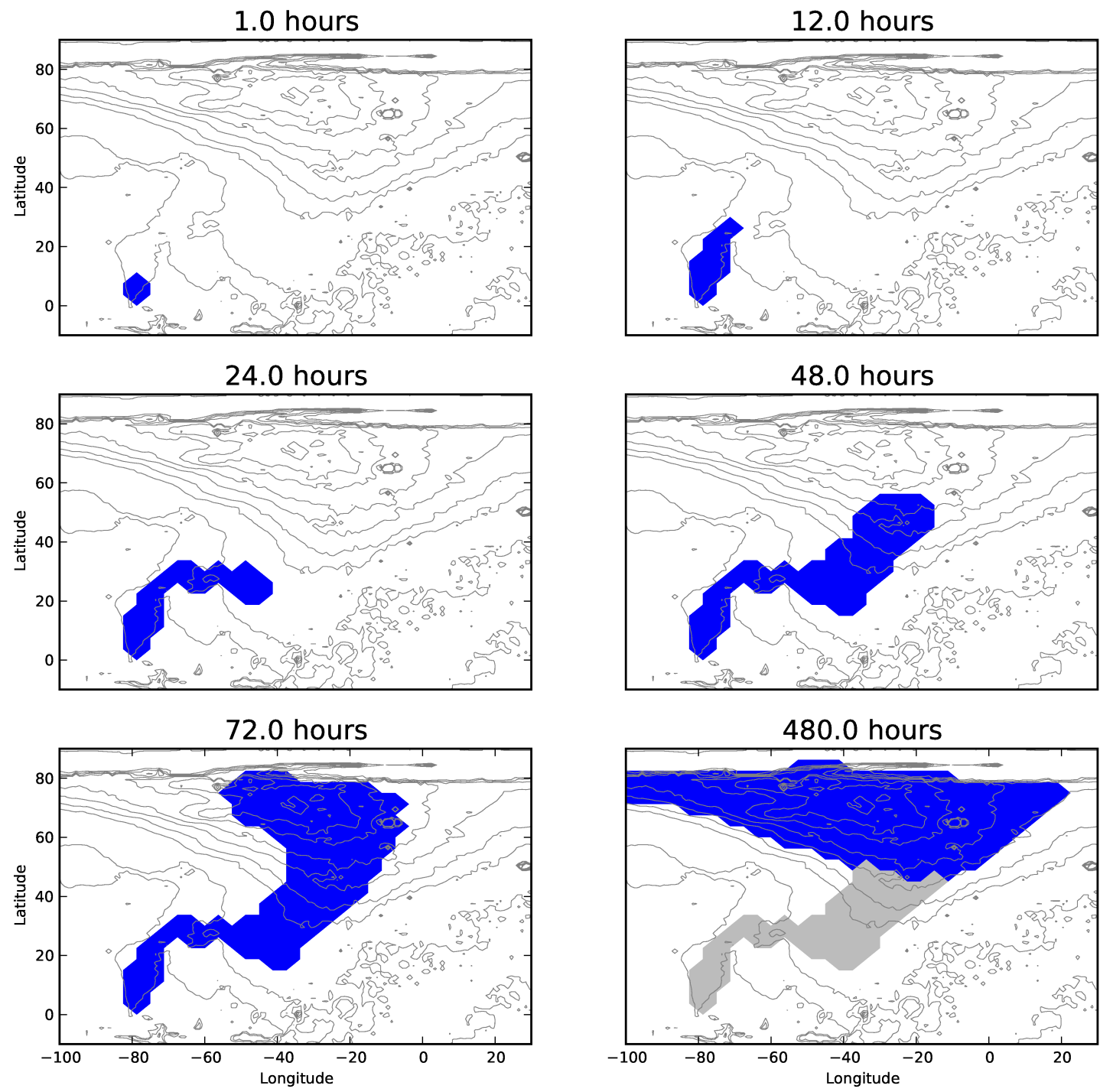

Figure 4: Time lapse of the runoff of the outflow channel event occuring in Echus Chasma, and flowing from Kasei Vallis down to the Northern Plains main topographic depression. The blue area corresponds to the position of the flow. The grey color was used to represent the 'wet' regions where the flow passed through but did not accumulate. 


\subsection{Description of the flow}

A volume of $10^{6} \mathrm{~km}^{3}$ of liquid water is released at the discharge rate of $1 \mathrm{~km}^{3} \mathrm{~s}^{-1}$. It takes approximately 1.1 martian days for the liquid water to travel from the source of the flow (in Echus Chasma, at $\sim 4^{\circ} \mathrm{N},-79^{\circ} \mathrm{E}$ ) to the end of Kasei Vallis (at $\sim 30^{\circ} \mathrm{N},-45^{\circ} \mathrm{E}$ ), and 1.5 more days for the same flow to reach the main topographic depression of the northern plains (at $\sim 70^{\circ} \mathrm{N},-30^{\circ} \mathrm{E}$ ). This corresponds, respectively, to mean flow speeds of $\sim 30 \mathrm{~m} \mathrm{~s}^{-1}$ and $\sim 16 \mathrm{~m} \mathrm{~s}^{-1}$, which are consistent with the two endmembers values shown in Figure 1 .

After $\sim 11$ days, the source of ground water (located in Echus Chasma) becomes inactive. Eventually, it takes approximately 20 martian days in this scenario for the liquid water that has erupted in Echus Chasma to form a stable lake in the lowest part of the Northern Plains. This lake extends over an area of 4.2 millions of $\mathrm{km}^{2}$ $(\sim 2.9 \%$ of the global surface area of Mars), has a mean depth of $\sim 240$ meters and a peak depth of $\sim 600$ meters. Some water ( $\sim 20$ centimeters) is left at locations with latitude $<50^{\circ} \mathrm{N}$ to account for the wet ground and the water possibly trapped in the topographic depressions.

The fate of the outflow channel formation event can be divided into two main parts:

1. During the first $\sim 500$ days following the event, the 'Warm Phase', an intense hydrological cycle takes place. The end of this phase approximately coincides with the time when the Northern Plains lake becomes fully covered by an ice layer.

2. During the following $\sim 10^{5}$ martian years, the martian climate is controlled by a weak and cold water cycle. It takes approximately the first $4 \times 10^{3}$ years (as predicted by simple energy-balanced models; Kreslavsky and Head 2002) for the lake to be entirely frozen, and the rest to sublimate the lake completely and move the ice to its positions of equilibrium, assuming no ice gets buried below a lag deposit or gets transported through glacier flows.

\subsection{The Warm Phase}

As soon as the simulation starts, the warm $300 \mathrm{~K}$ liquid water released in Echus Chasma evaporates efficiently following equation 9 , while flowing over the Northern Plains slopes. At the locations reached by the flow, which represent $\sim 11$ million $\mathrm{km}^{2}(\sim 7.5 \%$ of the global surface area of Mars), the evaporation rate can reach $\sim 10^{-3} \mathrm{~kg} \mathrm{~m}^{2} \mathrm{~s}^{-1}$ for tens of days. Figure 5 (left) shows the mean evaporation rate for the $4.2 \times 10^{6} \mathrm{~km}^{2}$ Northern Plains stable lake formed by the outflow channel 

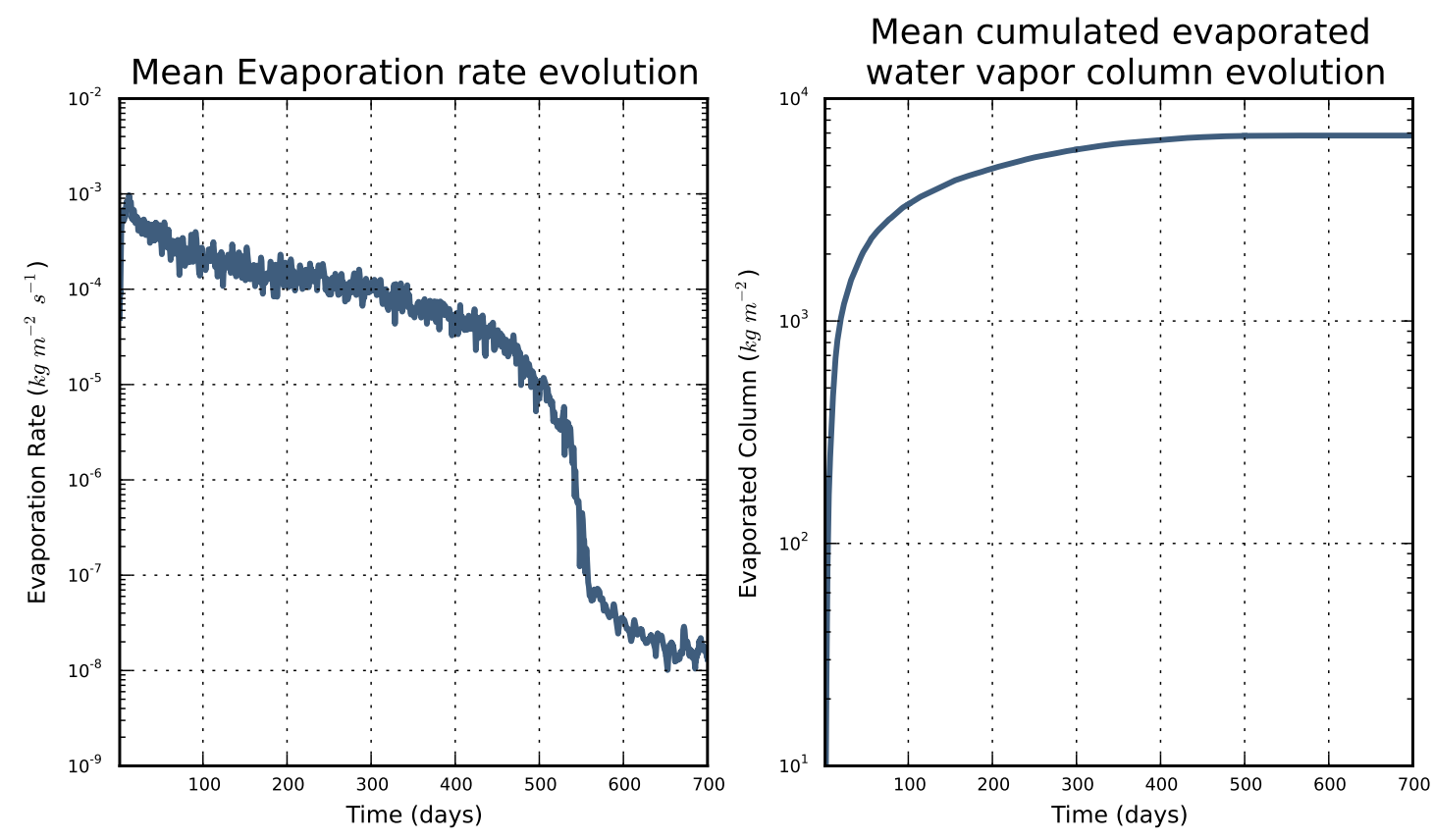

Figure 5: Mean lake evaporation rate evolution (left) and mean cumulative evaporated liquid water from the lake (right). The right curve is the cumulative integral over time of the left curve. 
flood accumulation.

During the 500 days following the event, a global precipitable water amount of $\sim 23$ centimers is evaporated by the liquid water flow. Evaporation of the lake accounts for $96 \%$ of this amount (blue region in Figure 4, after 480 hours) and $4 \%$ by the evaporation of the transient flow (grey region in Figure 4, after 480 hours). This amount of cumulative evaporation corresponds to $\sim 3.4 \%$ of the initial volume of water ejected by the ouflow event, which is approximately 0.7 times the amount of evaporated water that would be expected if the extra thermal heat (compared to $273 \mathrm{~K}$ ) of the $300 \mathrm{~K}$ flow was simply converted into latent heat.

\subsubsection{Mechanisms warming the atmosphere}

As the water vapor starts to accumulate above the flow, the initially cold martian lower atmosphere soon reaches the water vapor saturation pressure. For instance, at 210 Kelvins, which is typically the mean surface temperature expected for a 0.2 bar atmosphere (Figure 2), the water vapor saturation pressure is $\sim 1.4$ Pascals and the mass mixing ratio at saturation in a 0.2 bar atmosphere is thereby $7 \times 10^{-5} \mathrm{~kg} / \mathrm{kg}^{-1}$. This situation leads to the early condensation of the water vapor, latent heat release and thus to the warming of the atmosphere. We identified this process as the dominant mechanism responsible for the warming of the atmosphere after an outflow event.

As the atmospheric temperatures increase, the capability of the atmosphere to retain water vapor also increases. The mass mixing ratio at saturation, namely $Q_{\text {sat }}$, can be written as follows:

$$
Q_{\text {sat }, \mathrm{H}_{2} \mathrm{O}}=\frac{P_{\text {sat, } \mathrm{H}_{2} \mathrm{O}}}{P_{\mathrm{CO}_{2}}+P_{\mathrm{sat}, \mathrm{H}_{2} \mathrm{O}}} \text {, with } P_{\text {sat }, \mathrm{H}_{2} \mathrm{O}}(T)=P_{\text {ref }} e^{\frac{L_{\mathrm{v}} M_{\mathrm{H}_{2} \mathrm{O}}}{R}\left(\frac{1}{T_{\mathrm{ref}}}-\frac{1}{T}\right)} \text {, }
$$

with $P_{\text {sat, } \mathrm{H}_{2} \mathrm{O}}$ the water vapor saturation pressure and $P_{\mathrm{CO}_{2}}$ the $\mathrm{CO}_{2}$ partial pressure, with $P_{\text {ref }}$ and $T_{\text {ref }}$ the pression/temperature of the triple point of water, respectively equal to 612 Pascals/273.16 Kelvins, $M_{, \mathrm{H}_{2} \mathrm{O}} \sim 1.8 \times 10^{-2} \mathrm{~kg} \mathrm{~mol}^{-1}$ the molar mass of water, and $L_{v} \sim 2.26 \times 10^{6} \mathrm{~J} \mathrm{~kg}^{-1}$ the latent heat of vaporization of liquid water. For low amounts of water, this relation simply becomes:

$$
Q_{\text {sat, } \mathrm{H}_{2} \mathrm{O}}(T) \sim \frac{P_{\text {ref }}}{P_{\mathrm{CO}_{2}}} e^{\frac{L_{\mathrm{v}} M_{\mathrm{H}_{2} \mathrm{O}}}{R}\left(\frac{1}{T_{\mathrm{ref}}}-\frac{1}{T}\right)} .
$$

Therefore, as the atmospheric temperatures increase, the atmosphere is also able to transport more and more water upwards. Thus, as time goes on, the 

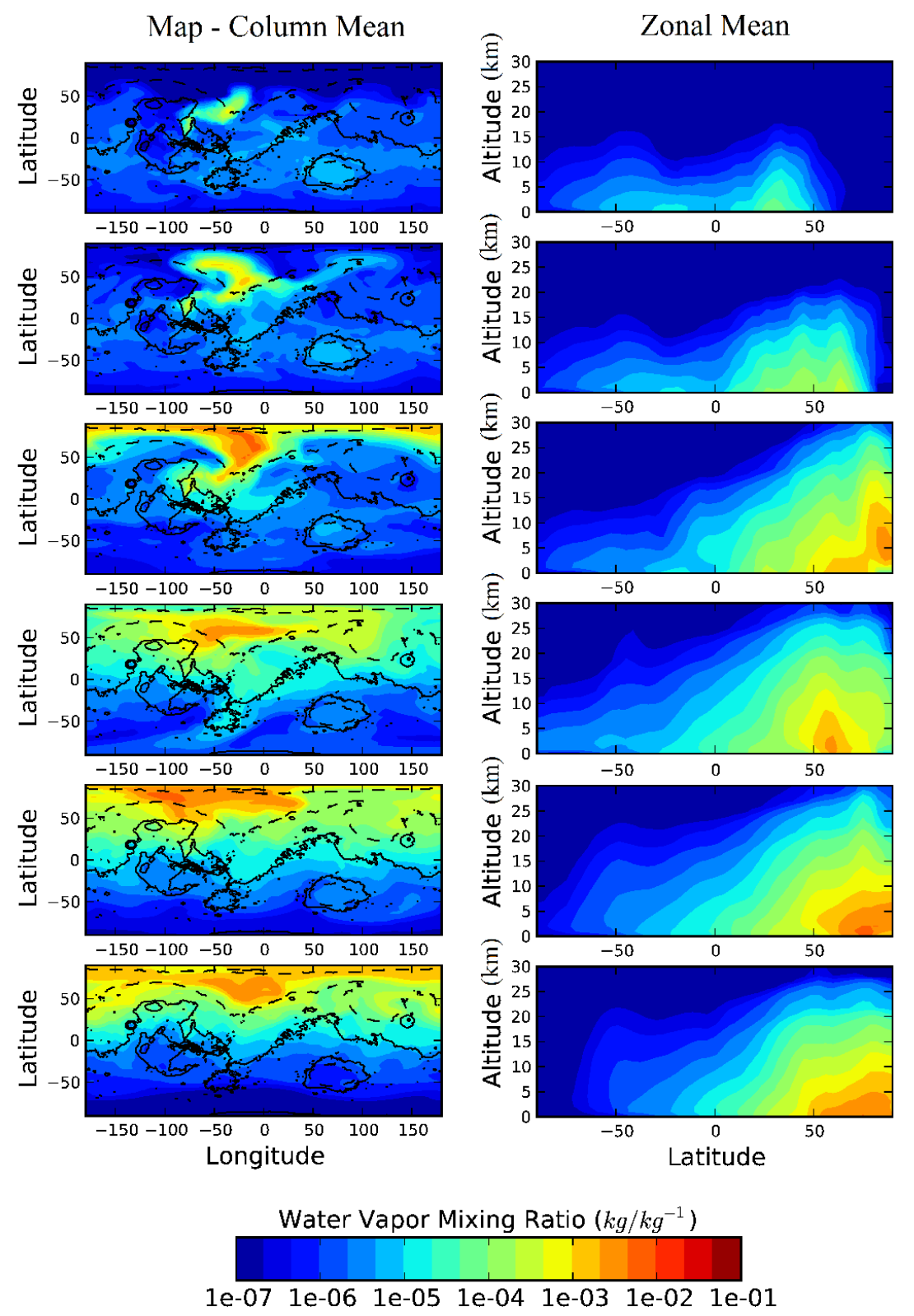

Figure 6: Time-lapse of the water vapor mixing ratio after (from the top to the bottom) $2.5 / 5 / 10 / 20 / 40 / 80$ days. The left panels show the map of the water vapor distribution (column mean mixing ratio); the right panels show the corresponding zonal mean distribution (water vapor mixing ratio) as a function of latitude $\left({ }^{\circ} \mathrm{N}\right.$ ) and altitude $(\mathrm{km})$. 
atmosphere becomes more and more warm and wet. As the atmospheric water vapor content increases, the absorption of the atmosphere in the infrared wavelength range (essentially due to the thermal emission of the warm outflow waters) increases and thus contributes to an additional warming of the atmosphere.

In total, during the warm phase (the first 500 days), the atmosphere (above the flow/lake) is directly warmed by the following processes (in decreasing order of importance): 1 . the condensation of the water vapor produced by the warm flow $(\sim 56 \%) ; 2$. the sensible heat exchanged between the flow/lake and the lowest atmospheric layer $(\sim 22 \%) ; 3$. the thermal infrared emission of the flow absorbed by the mixture of gaseous $\mathrm{CO}_{2} / \mathrm{H}_{2} \mathrm{O}(\sim 13 \%)$; and 4. the extra solar absorption resulting from the presence of water vapor excess, which has strong absorption lines in the solar domain $(\sim 9 \%)$;. The atmospheric solar absorption is particularly important in this scenario, because we chose the outflow channel event to start at $\mathrm{Ls}=5^{\circ}$ and thus to occur during the northern hemisphere spring and summer. Of course, all these processes reinforce and strengthen each other.

Figure 6 shows the spatial evolution of the water vapor atmospheric content. Initially, water vapor accumulates at low altitudes, in the regions where the liquid water flow is located. After a few days, the water vapor has reached much higher altitudes (up to $\sim 30 \mathrm{~km}$ ) through the aforementioned warming mechanisms and the convective adjustment scheme. Eventually, once the upper part of the atmosphere has become wet enough (typically after $\sim 10$ days in this scenario), the high altitude horizontal winds (around $\sim 15 \mathrm{~km}$ ) advect the water vapor into the neighbouring regions. After $\sim 50$ days, all the martian regions located above $\sim 50^{\circ} \mathrm{N}$ have become more or less wet, with a typical water vapor mean mass mixing ratio of $0.3 \%$.

Similarly, the impact of $\mathrm{H}_{2} \mathrm{O}$ condensation (and other additional warming sources) on atmospheric temperatures is shown in Figure 7. After $\sim 100$ days, at the peak of the outflow channel event, the atmospheric temperatures in the lower atmosphere $(0-5 \mathrm{~km})$ almost reach $280 \mathrm{~K},+90$ Kelvins above the regular temperature (peak above the lake) as calculated in the control simulation; the atmospheric temperatures in the higher parts of the atmosphere typically extend up to 230 Kelvins (at $10 \mathrm{~km}$ ) and to 170 Kelvins (at $25 \mathrm{~km}$ ), which are respectively $+50 \mathrm{~K}$ and $+25 \mathrm{~K}$ above the temperatures prescribed by the control simulation.

\subsubsection{The mechanisms cooling the flow}

After $~ 500$ days, which corresponds to the complete surface freezing of the outflow channel event water, the evaporation E produced by the stable lake (see 


\section{Atmospheric Temperatures Changes}

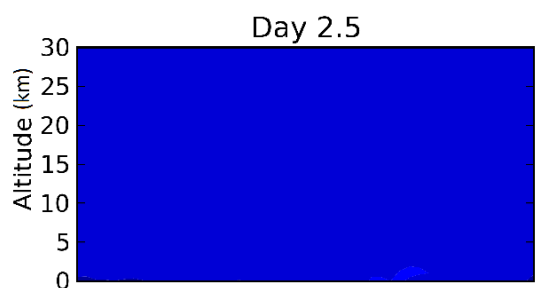

Day 20.0

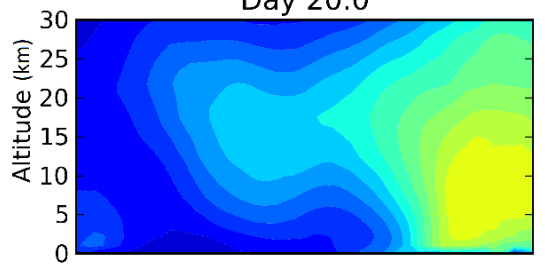

Day 80.0

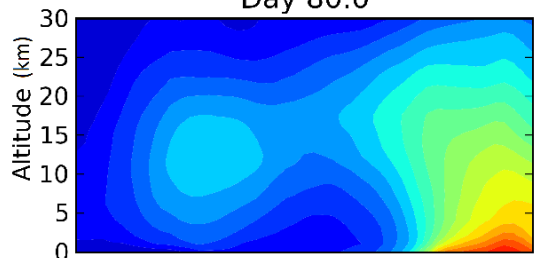

Day 180.0

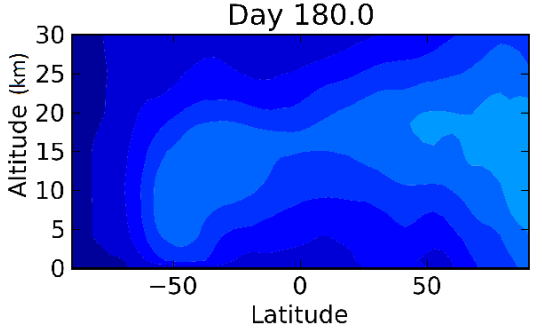

Day 5.0

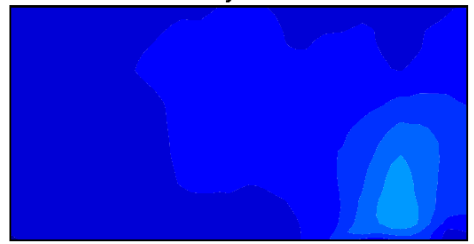

Day 40.0

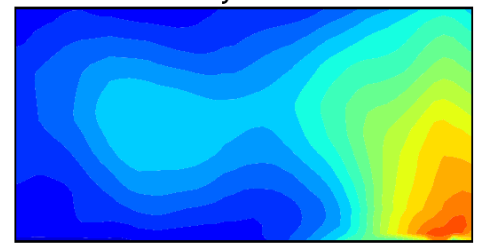

Day 100.0

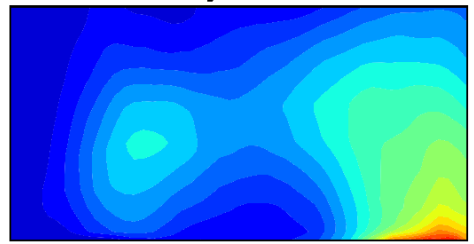

Day 220.0

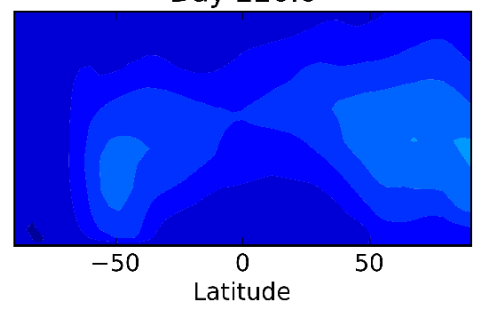

$\Delta T($ in $\mathrm{K})$

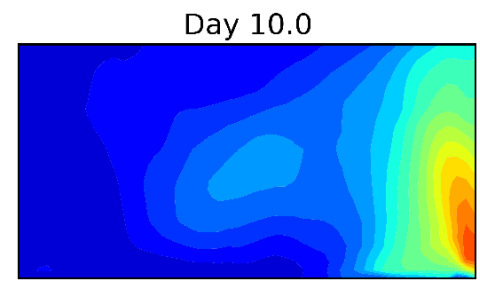

Day 60.0

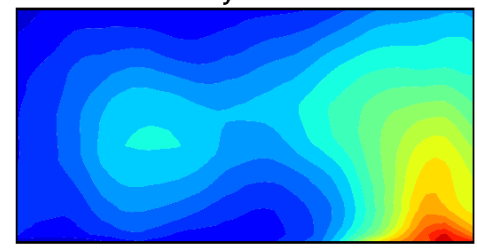

Day 140.0

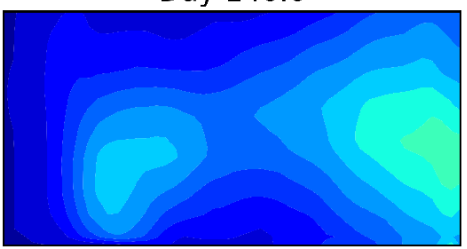

Day 260.0

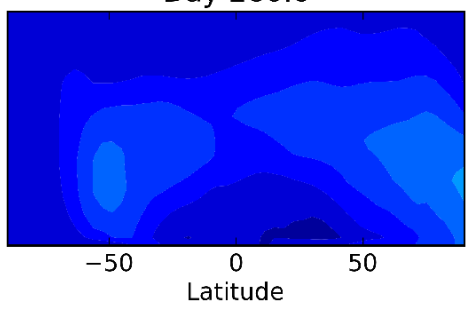

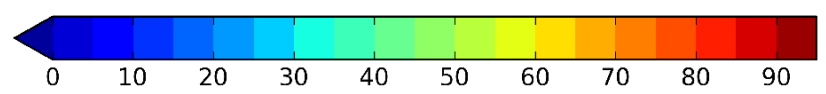

Figure 7: Time-lapse of the zonal mean cross-section atmospheric temperature difference between the reference simulation (with outflow) and the control simulation (without outflow) for the same surface pressure of 0.2 bar. 
Figure 57 suddenly reduces (by almost 3 orders of magnitude). To a first order, we have in fact:

$$
E \propto Q_{\text {sat }}(T) \propto e^{-\frac{\alpha}{T}},
$$

with $\alpha=\frac{L_{\mathrm{sub}} M_{\mathrm{H}_{2} \mathrm{O}}}{R}$ and $L_{\text {sub }}$ the latent heat of sublimation of water ice. The evaporation rate $\mathrm{E}$ has thereby a strong dependence on temperature. This is why the drop in temperature associated with the surface freezing of the Northern Plains lake is responsible for the sudden decrease of evaporation visible in Figure 5 (also seen through the latent heat surface flux in Figure 8). This drop in evaporation defines the end of the 'warm phase', which includes the decrease of the water vapor content, the atmospheric temperatures and the precipitation events (see Figure 12).

There are several physical processes that are responsible for the cooling of the flow, leading to its solidification as ice. Figure 8 shows the relative importance of the different thermal heat losses by the Northern Plains lake, from the beginning of the event to one martian year later. For the first 500 days, the main cooling surface fluxes are the latent heat loss $\left(420 \mathrm{~W} \mathrm{~m}^{-2}, 43.3 \%\right)$, the sensible heat loss $\left(190 \mathrm{~W} \mathrm{~m}^{-2}, 19.6 \%\right)$, the radiative thermal emission loss $\left(280 \mathrm{~W} \mathrm{~m}^{-2}, 28.8 \%\right)$ and the ground conduction loss $\left(8 \mathrm{~W} \mathrm{~m}^{-2}, 0.8 \%\right)$. Some other surface fluxes related to the $\mathrm{CO}_{2}$ ice sublimation by the warm flow $\left(13 \mathrm{~W} \mathrm{~m}^{-2}, 1.3 \%\right)$ and the cooling of the lake by the melting of the falling snow $\left(60 \mathrm{~W} \mathrm{~m}^{-2}, 6.2 \%\right)$ also contribute to the cooling of the outflow waters. In total, the average cooling flux of the outflow waters for the warm phase (first 500 days) is $\sim 970 \mathrm{~W} \mathrm{~m}^{-2}$.

For large outflow channel formation events like the one described in this section, the sublimation of the seasonal carbon dioxide ice deposit represents a small fraction of the heat loss. Nonetheless, smaller outflow channel events $\left(5 \times 10^{3} \mathrm{~km}^{3}\right.$ for example (Andrews-Hanna and Phillips, 2007)) flowing on the Northern Plains slopes may be deeply affected by the energy gap required to sublimate the $\mathrm{CO}_{2}$ ice seasonal deposit. For a 0.2 bar atmosphere, the control simulations show, for example, that the $\mathrm{CO}_{2}$ ice seasonal deposit reaches a yearly average of $\sim 300 \mathrm{~kg} \mathrm{~m}^{-2}$ from the North Pole down to $30^{\circ} \mathrm{N}$ latitudes.

Two radiative processes may counteract the cooling of the flow: 1) the absorption of solar radiation and 2) the greenhouse effects (of the atmosphere and of the clouds).

1. We chose in this scenario to start the outflow channel event at Ls $=5^{\circ}$ in order to maximize the role of solar absorption. The peak of the event (between $\sim 0$-300 days, Ls $\sim 5-165^{\circ}$ ) was therefore chosen to overlap with the peak of insolation in the Northern hemisphere, which is a maximum of $\sim 170$ days after 


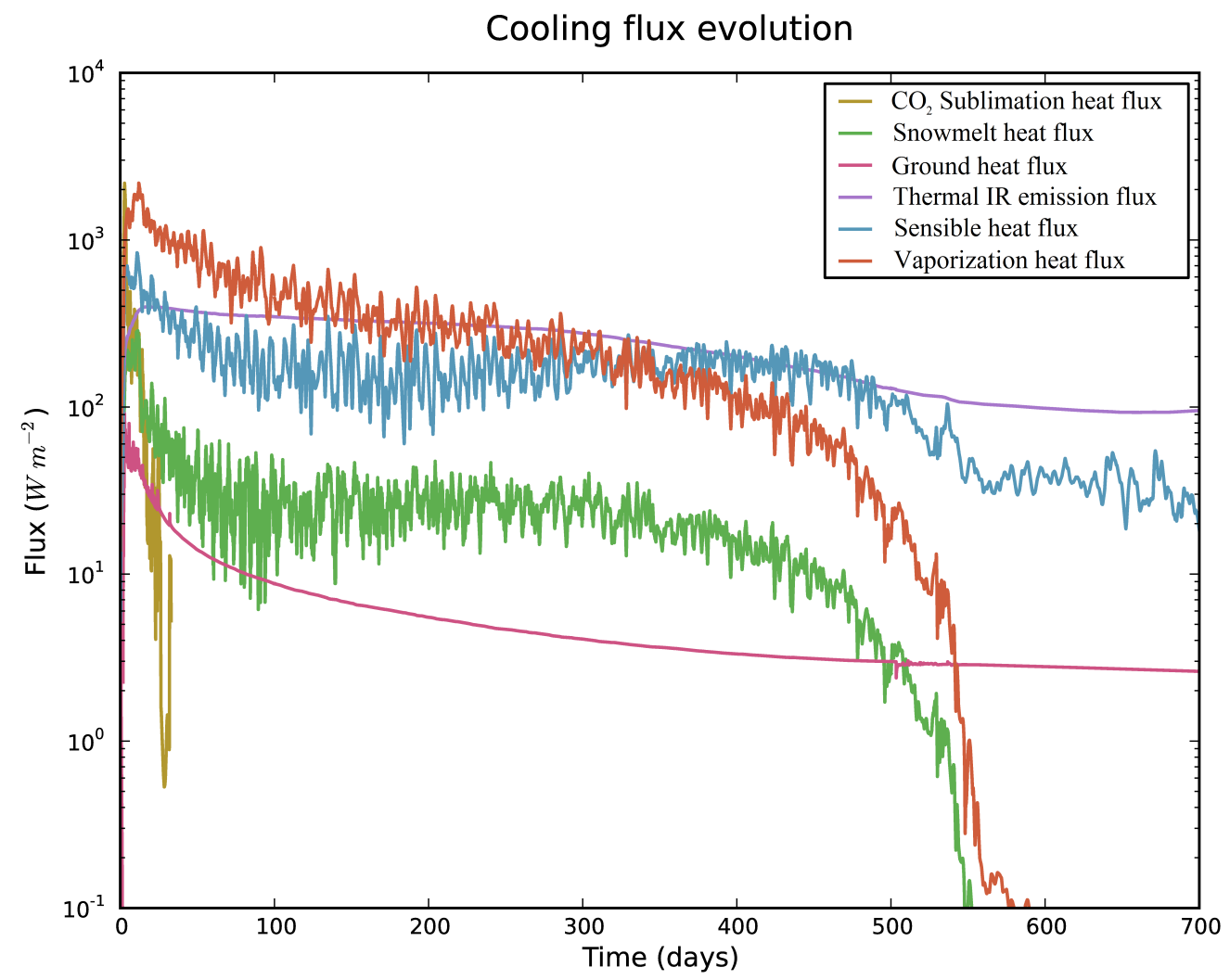

Figure 8: Surface cooling heat fluxes evolution averaged over all the Northern Plains lake grid cells in the $\mathrm{P}_{\text {surf }}=0.2$ bar reference simulation. We note here that, depending on the nature and the intensity of a given outflow channel formation event, each of these fluxes can potentially become dominant. 


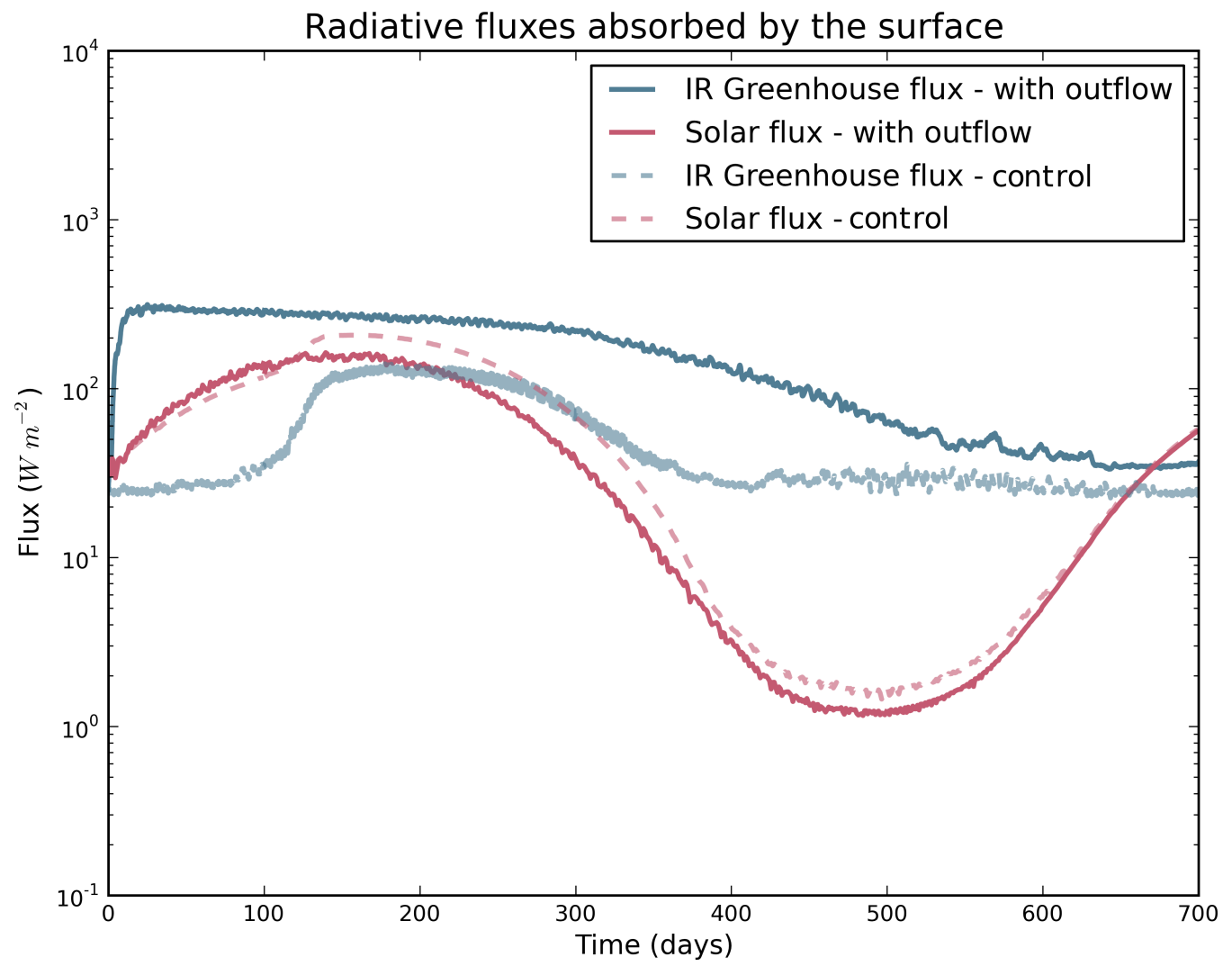

Figure 9: Evolution of the radiative fluxes absorbed by the surface and averaged over all the Northern Plains lake grid cells. Solid lines refer to the solar flux (in red) and the thermal infrared (in blue) for the reference simulation. Dashed lines correspond to the control simulations. For better visibility, we filtered diurnal waves from the absorbed solar fluxes using a 1 day running average. 

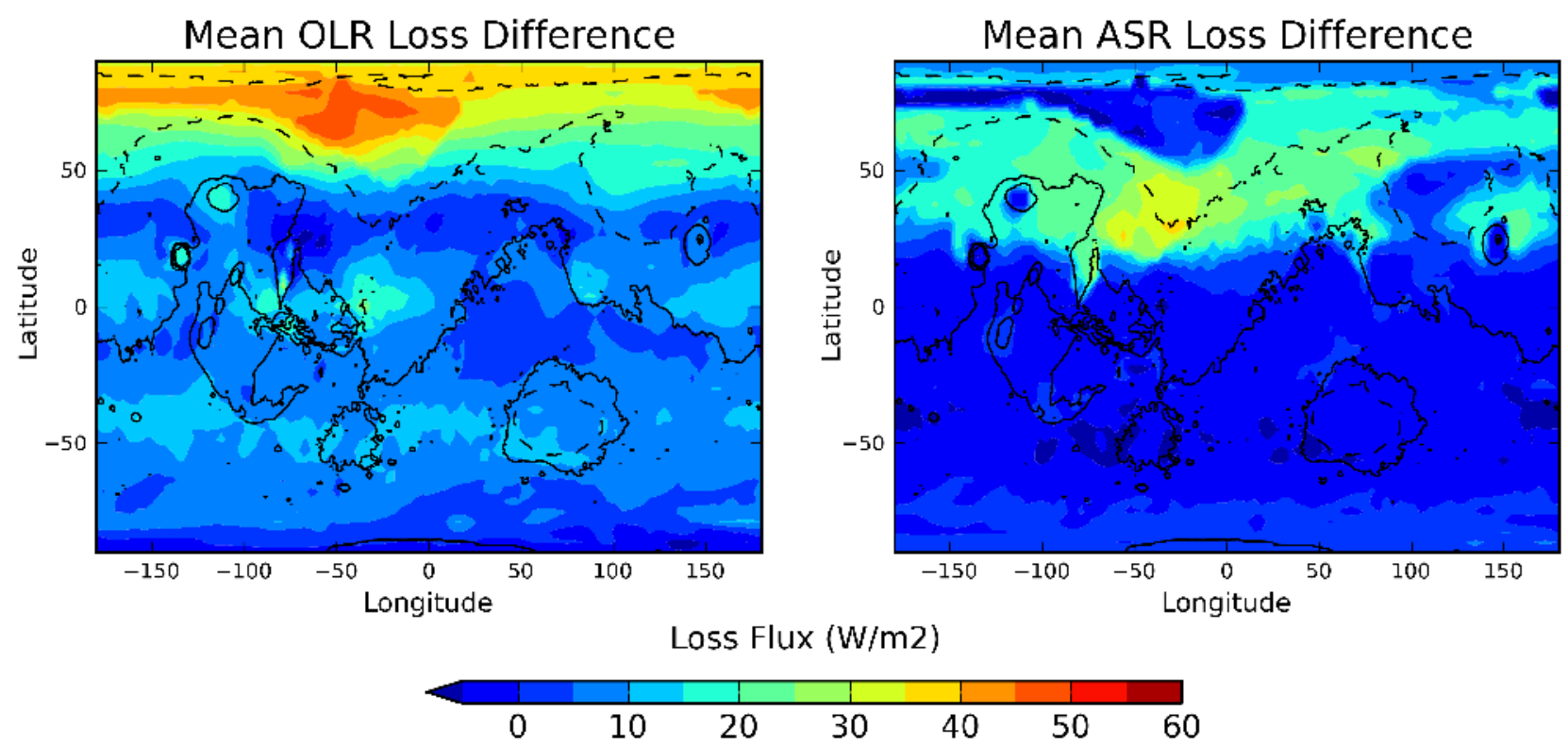

Figure 10: Mean Outgoing Longwave Radiation (OLR, left) and Absorbed Solar Radiation (ASR, right) loss during the warm phase, for the reference simulation, and relative to the control simulation performed for the same surface pressure.

the event $\left(\mathrm{Ls}=90^{\circ}\right)$. There are three factors that need to be taken into account in the solar absorption processes: absorption by water vapor, albedo changes and clouds. For this reference simulation, compared to the control simulation, these three effects more or less compensate at the location of the flow. The increase of the solar absorption due to the low albedo of liquid water ( 0.07 compared to 0.2 for the bare ground and 0.5 for the remaining $\mathrm{CO}_{2}$ ice seasonal cover) and due to the absorption by water vapor are more or less balanced by the reflection of the cloud cover, which can reach on average a coverage of $80 \%$ during the first 500 days above the lake (Figure 11). Most of these water clouds are located at low altitude (Figure 11). During the warm phase, the lake absorbs a solar flux of $\sim 67 \mathrm{~W} \mathrm{~m}^{-2}\left(\sim 16 \mathrm{~W} \mathrm{~m}^{-2}\right.$ less than the control run, see Figure 9) and the atmosphere (essentially the troposphere) $\sim 20 \mathrm{~W} \mathrm{~m}^{-2}\left(\sim 12 \mathrm{~W} \mathrm{~m}^{-2}\right.$ more than the control run). This corresponds to an average absorption of $65 \%$ of the available incoming solar flux ( $\sim 135.6 \mathrm{~W} \mathrm{~m}^{-2}$ for the first 500 days).

2. The downward thermal infrared emission from the atmosphere and the 

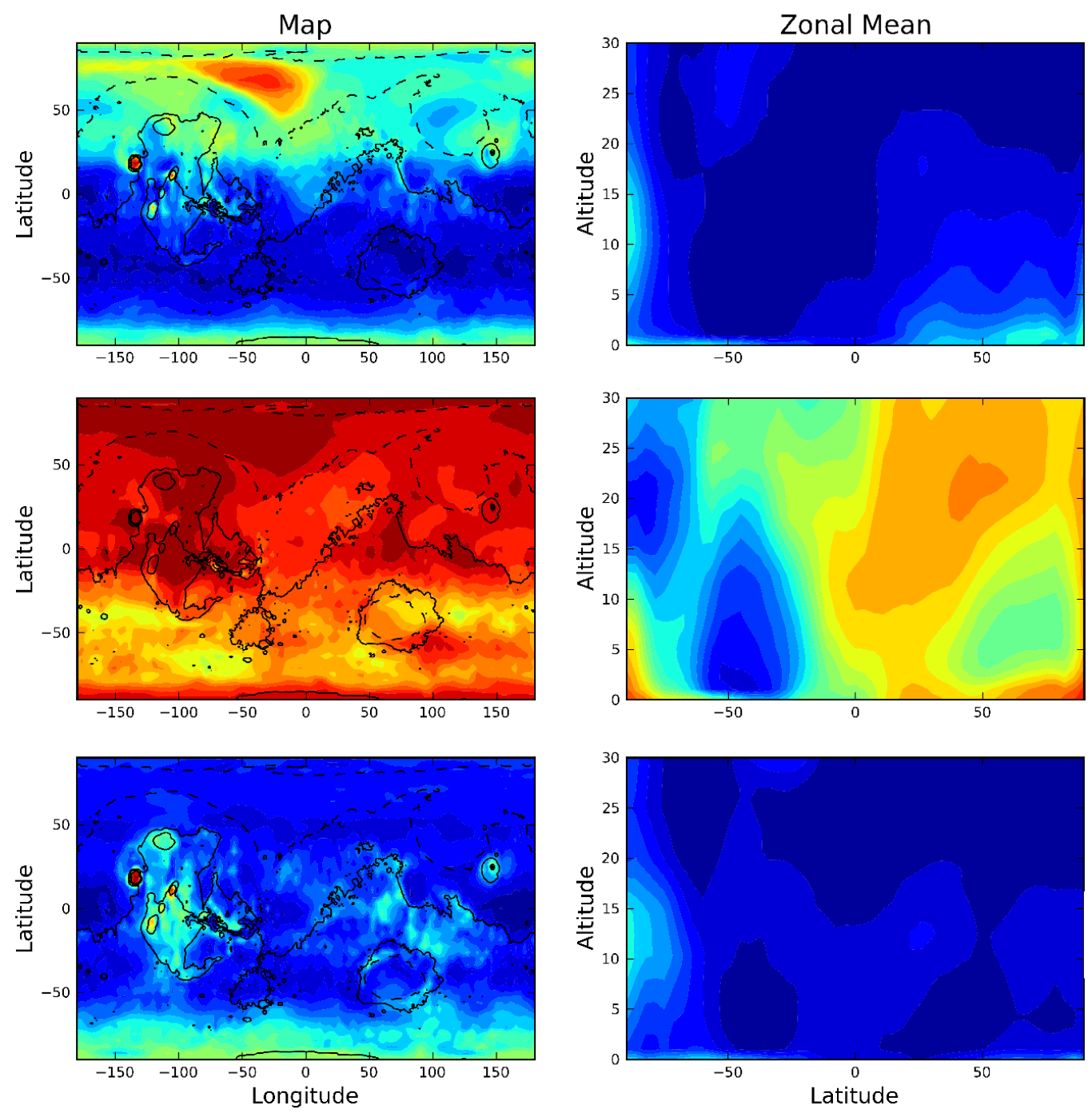

Cloud coverage percentage

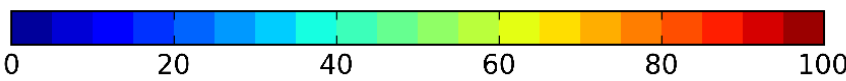

Figure 11: One year average of the cloud coverage following the outflow event. The first row corresponds to the map and the zonal mean cross-section of the reference simulation. In the second row, precipitation was removed (see section 7.2). The third row is for the control simulation. 
clouds is the dominant warming flux (see Figure 9). On average, during the warm phase, this greenhouse effect brings $\sim 210 \mathrm{~W} \mathrm{~m}^{-2}$ to the lake $\left(+150 \mathrm{~W} \mathrm{~m}^{-2}\right.$ more than the control run). The main source of thermal infrared emission surface heating comes from the gaseous atmosphere itself, which can reach up to $\sim 280 \mathrm{~K}$ (above the lake) for the first $5 \mathrm{~km}$, at the peak of the event.

In total, both solar and infrared heating counterbalance only $\sim 30 \%$ of the cooling of the flow, and are thus unable to sustain the perturbation generated by the ouflow channel. We note here that the radiative effect of $\mathrm{H}_{2} \mathrm{O}$ clouds during the warm phase is approximately neutral or at least very limited (only $+7 \mathrm{~W} \mathrm{~m}^{-2}$ ) above the Northern Plains lake, with $+23 \mathrm{~W} \mathrm{~m}^{-2}$ of greenhouse warming and $-17 \mathrm{~W} \mathrm{~m}^{-2}$ due to the reflection of the sunlight.

\subsubsection{The mechanisms cooling the atmosphere}

One of the main results of our work is that outflow channel events are not able to sustain warm conditions. We present here the two processes that act efficiently together to cool down the atmosphere after outflow events.

1. In the time following catastrophic outflow channel events like the one described in this section, the atmosphere above the flow warms very quickly. In our reference simulation, 10 days after the beginning of the event, the temperature in the lower atmosphere $(0-5 \mathrm{~km})$ above the lake increases by almost 90 Kelvins. During the first 500 days after the event, because of this significant warming, the flow and the atmosphere just above it contribute to an extra thermal infrared emission loss to space of $38 \mathrm{~W} \mathrm{~m}^{-2}$ compared to the control simulation. Yet the amount of energy lost by the lake and the atmosphere above represent only $\sim 11 \%$ of the extra total cooling to space. Figure 7 shows that, as the atmosphere gets warmer in the regions of the flow, high altitude winds around $\sim 15 \mathrm{~km}$ advect the heat to the neighbouring areas (in particular into the Northern Plains). This increases the surface of the emissions and therefore strengthens the cooling.

Figure 10(left) shows the regions of the planet responsible for the extra thermal emission to space. Globally, during the warm phase (the first 500 days), the planet loses $\sim 10 \mathrm{~W} \mathrm{~m}^{-2}$. One third of the emissions are due to the regions of latitude $>50^{\circ} \mathrm{N}$. During the warm phase, the most important mechanism of cooling is the thermal infrared emission, enhanced by the advection processes.

2. Interestingly, another important cooling mechanism is the decrease of solar absorption due to the increase of surface albedo that follows the outflow channel event. In fact, the precipitation caused by the event, essentially in the form of snowfall (see Figure 12), leaves ice (see Figures 13 and 10) over an area of 

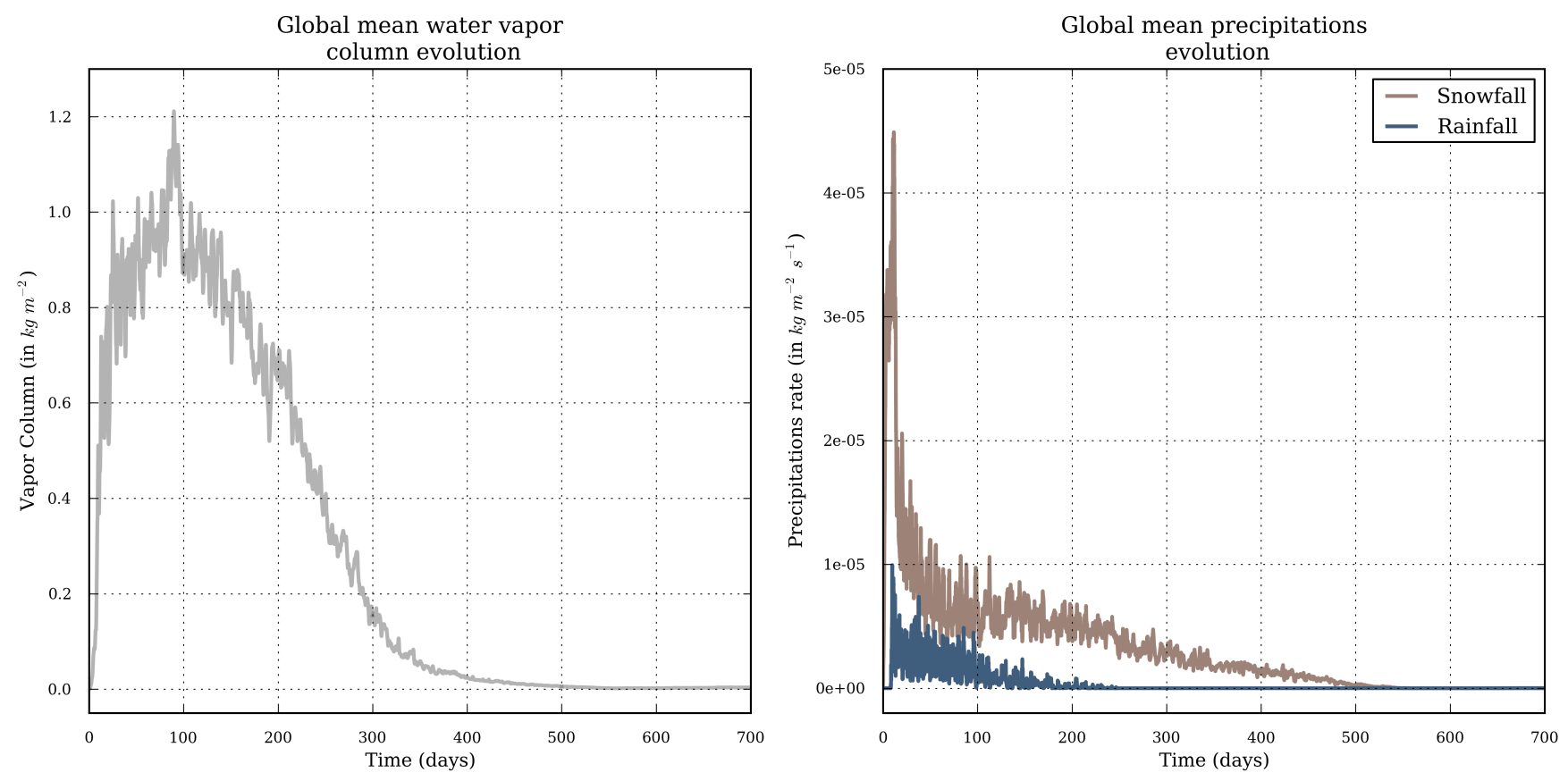

Figure 12: Evolution of the global mean water vapor column (left) and the precipitation (right), during the year following the outflow channel reference event.

$\sim 30 \times 10^{6} \mathrm{~km}^{2}$ that reflect an important part of the sunlight $\left(\sim 21.5 \mathrm{~W} \mathrm{~m}^{-2}\right)$. In total, during the warm phase and compared to the control simulation, the decrease of solar absorption contributes to a global equivalent extra cooling of $\sim 4.5 \mathrm{~W} \mathrm{~m}^{-2}$, which represents half of the infrared emission loss to space.

The large amount of water vapor released after the outflow channel event condenses very quickly in the atmosphere, forming clouds that are mostly located in the area of the flow and of the resulting lake (see Figure 11). In total, for the reference simulation, the clouds have a slight positive effect of $+1.3 \mathrm{~W} \mathrm{~m}^{-2}$ $\left(+2.3 \mathrm{~W} \mathrm{~m}^{-2}\right.$ of greenhouse effect and $-1.0 \mathrm{~W} \mathrm{~m}^{-2}$ of solar reflection).

\subsubsection{Consequences on the water cycle and the precipitation}

The maximum total amount of water vapor that is carried by the atmosphere during the event (GEL of $1.2 \mathrm{~mm}$ at the peak) remains limited by comparison to the cumulative total amount of precipitable water generated (GEL of $230 \mathrm{~mm}$ ). 
It represents only $\sim 0.5 \%$ of the cumulative evaporated water vapor produced by the entire ouflow channel event during the first 500 days. Figure 12 (left) shows the global mean atmospheric water vapor content (column mass in $\mathrm{kg} \mathrm{m}^{-2}$, and also GEL in $\mathrm{mm}$ ). It peaks at $\sim 100$ days and considerably decreases from $\sim 200$ days to $\sim 500$ days.

The fact that the atmosphere is not able to accumulate more than $\sim 1.2 \mathrm{~kg} \mathrm{~m}^{-2}$ (globally) and $\sim 50 \mathrm{~kg} \mathrm{~m}^{-2}$ (locally, just above the warm lake) has one main consequence: the atmosphere does not manage to carry enough water vapor far enough from the lake to create precipitation in regions of interest (West Echus Chasma Plateau in particular). The typical lifetime of the atmospheric water vapor is in fact $\sim 0.5$ days

Rainfall, which represents a very small fraction $(\sim 10 \%)$ of the precipitation (Figure 12), occurs only above the Northern Plain lake, because this is the only location of Mars where atmospheric temperatures exceed (up to $10 \mathrm{~km}$ ) the temperature of the triple point. Outside the lake, the only mechanism of precipitation is snowfall. Approximately $50 \%$ of the snow falls back directly on the flow/lake. The rest of the precipitation (the $50 \%$ remaining) is essentially confined in the northern regions. Figure 13 shows the map of the deposited ice field (generated by precipitation) after a simulation of one martian year. The fraction of this ice that is melted after an outflow event is very limited (see Figure 17), because 1) most of the thermal perturbation has been dissipated by advection/cooling to space processes after $\sim 200$ days, 2) the remaining water vapour abundance after these 200 days is too low to trigger a significant greenhouse warming (as found by Kite et al. 2011a) and 3) the ice field itself raises the albedo of the surface and thus acts as a very efficient climatic cooling agent.

In summary, the short-term climatic impact of outflow channel formation events seems very limited. For a 0.2 bar atmosphere, an outflow channel event of $10^{6} \mathrm{~km}^{3} / 300 \mathrm{~K}$ leads to the formation of a lake (located in the Northern Plains main topographic depression) that triggers a warm period that lasts for $\sim 500$ days, which coincides approximately with the complete surface freezing of the water in the lake. Such events leave globally $\sim 6.5 \times 10^{3} \mathrm{~km}^{3}$ of water ice/snow $(0.65 \%$ of the initial outflow reservoir) and are able to melt $\sim 80 \mathrm{~km}^{3}(0.008 \%$ of the initial reservoir; $1 \%$ of the deposited precipitation). Because the outflow events do not manage to warm the atmosphere enough, water vapour stays confined to the regions neighbouring the lake (essentially in the Northern Plains) and therefore precipitation (mostly snowfall) and melting only occur in the lowland regions.

The long-term climatic impact of the ice-covered lake is discussed in the next section. 


\subsection{The Cold Phase}

After 500 martian days, the surface of the Northern Plains lake is completely covered by ice. Temperatures, water vapor content and precipitation all decrease. Because the area of high albedo ice deposits is larger than in the control simulations, the mean surface temperatures extend even lower than before the ouflow event ( $-2 \mathrm{~K}$ for the global annual surface temperatures of the 0.2 bar reference simulation, and compared to the control simulation).

Using the extrapolation scheme presented in section 3.3.1, we estimated that the released water was completely frozen after $\sim 4 \times 10^{3}$ martian years. This corresponds to the full solidification of the water to ice at the location of the main Northern Plains topographic depression (which is the deepest point of the lake). After 500 years, more than $70 \%$ of the lake (in area) is frozen, from the surface to the top of the regolith. We note that the ground thermal flux (Clifford and Parker, 2001) during the Late Hesperian era was one order of magnitude too low (at best) to be able to increase the lifetime of the $\sim 500 \mathrm{~m}$ deep lake.

In our simulations, $\sim 10$ years after the beginning of the lake-forming event, the mean ice thickness over the lake is $\sim 25$ meters. The annual mean conduction heat flux for this ice thickness is $\sim 10 \mathrm{~W} \mathrm{~m}^{-2}$. The annual mean solar/IR fluxes absorbed by the ice are $\sim 53 / 57 \mathrm{~W} \mathrm{~m}^{-2}$ by comparison, $110 \mathrm{~W} \mathrm{~m}^{-2}$ in total. Under these conditions, the thermal conduction flux represents less than $10 \%$ of the total heat flux received by the surface at the location of the lake. Moreover, because the temperature profile oscillates annually, in the first 5 meters (typically) of the ice cover, from positive values (summer season) to negative values (winter season), the heat conduction from the liquid water to the surface is mainly returned during the winter seasons. Yet, the water cycle in this cold phase is essentially controlled by the summer seasons, because sublimation rates are several orders of magnitude higher than during the winter seasons (see section 3.3.1 for discussion). Thus, after a few years (typically around 10), the climatic effect of the lake becomes, to a first order, the same as simply placing a comparable-sized body of ice in the Northern Plains. During these 10 years, ice transportation/water vapor cycle/precipitation is very limited by comparison to the warm phase and do not play any significant role in the ice field position.

Within the lifetime of the liquid water lake, the ice field position evolution is completely controlled by the $\sim 4 \times 10^{3}(-10)$ years of the water cycle forced by the sublimation of the large body of non-stable ice.

Each year, during Northern summer, $\sim 20 \mathrm{~mm} \mathrm{year}^{-1}$ of lake ice sublimes to condense elsewhere and approximately $30 \%$ of it is transported away from 


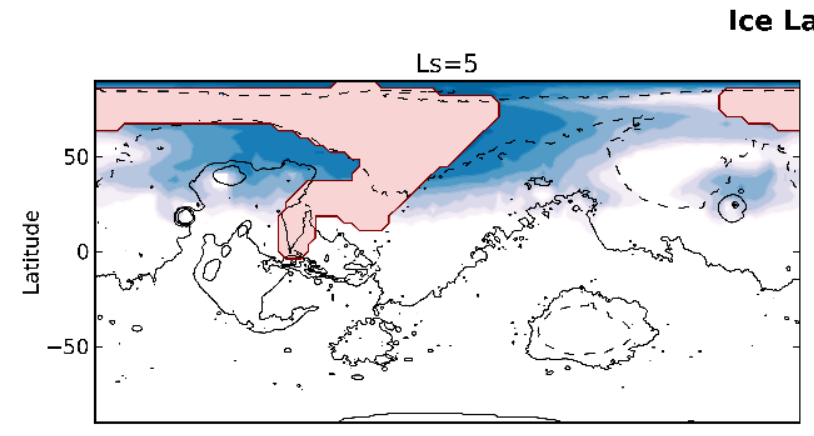

Ice Layer Deposit
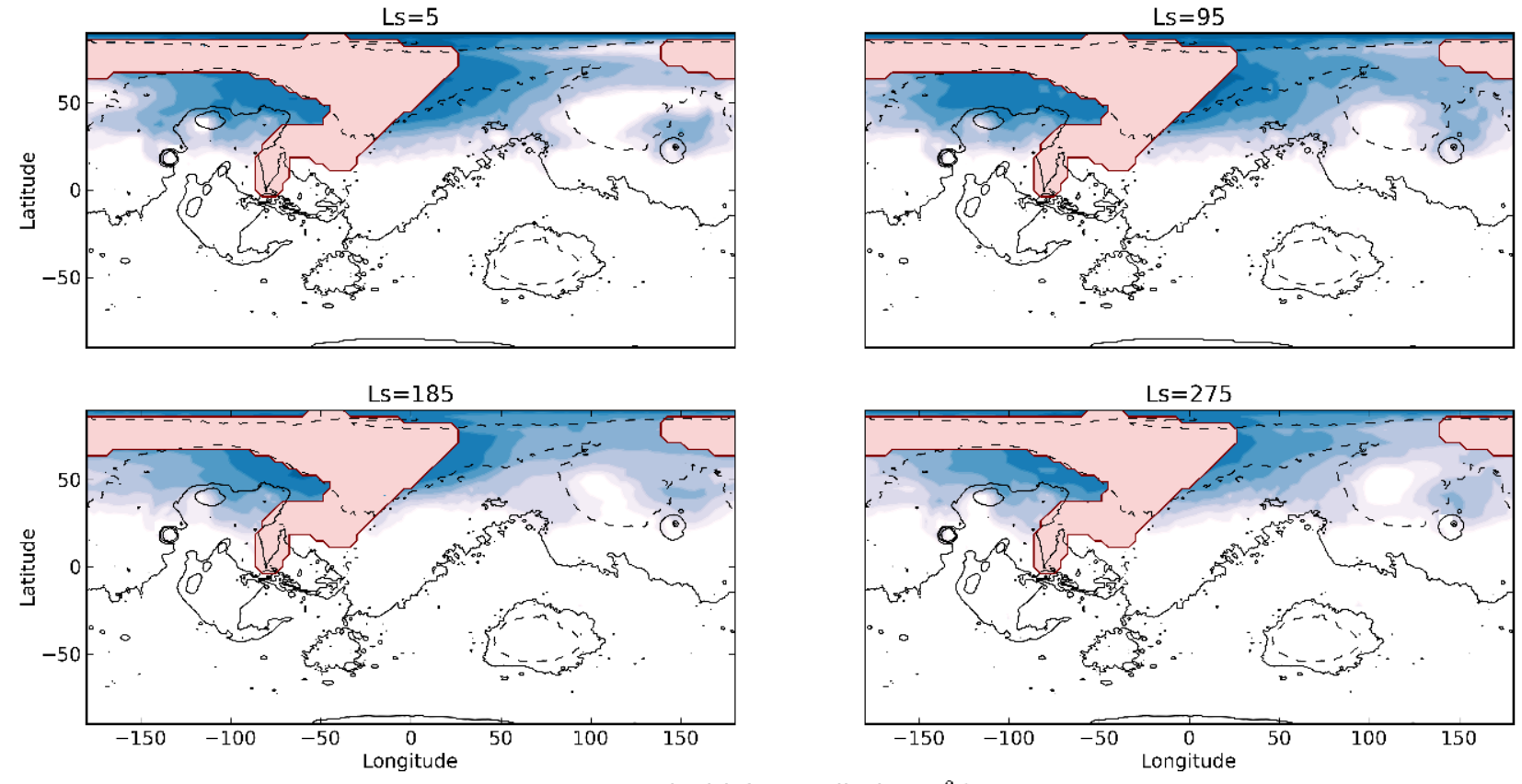

Deposit thickness (in $\mathrm{kg} / \mathrm{m}^{2}$ )

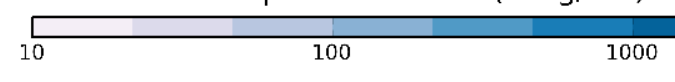

Figure 13: Ice deposit field obtained 1 martian year after the beginning of the event, for four different start dates $\left(\mathrm{Ls}=5^{\circ}, 95^{\circ}, 185^{\circ}, 275^{\circ}\right)$. The first figure (on the top left corner) corresponds to the reference simulation and starts at Ls $=5^{\circ}$. We use the pink color for the regions where the flow passed through on its way to the lake. 
the lake. Progressively, the water vapor produced during the summers migrates southward and - through the mechanism of adiabatic cooling - condenses on the regions of high altitudes and low latitudes. The lifetime of the frozen lake predicted by our simulations is $\sim 7 \times 10^{4}$ martian years.

The evolution of the ice field through the phases that follow the outflow channel reference event are shown in Figure 14. After $\sim 10^{5}$ martian years, the outflow channel water is located more or less exclusively in the highland regions. During this cold phase $\left(\sim 10^{5}\right.$ martian years $)$, some ice appears stable in the region of West Echus Chasma Plateau, due to the uninterrupted supply of ice coming from the northern parts of the planet. This snow deposit is produced by the adiabatic cooling of the ascending air masses that provoke the condensation of the water vapor initially generated by the sublimation of the Northern Plains ice field.

Some water ice is also transported to the drainage regions of Alba Patera, Hecates Tholus and Ceraunius Tholus but this might not be a critical factor since our model already predicts that ice deposits should be stable in these regions (Wordsworth et al. 2013, Figure 2 this work) and therefore available for either seasonal snowmelt or ground melting.

In spite of this, because the global surface albedo is increased during that period, global temperatures are much lower than before the outflow event, making snowmelt difficult.

We note here that we did not take into account the flow of the ice on the Northern Plains slopes. This could significantly increase the lifetime of the lake located in the main topographical depression and thus the lifespan of the snow deposited in non-stable locations (in particular in West Echus Chasma Plateau area). However, at these temperatures and over these timescales, ice is unlikely to flow significantly (Fastook et al., 2012; Fastook and Head, 2014, 2015). In addition, we did not take into account the formation of a possible lag deposit (Kreslavsky and Head, 2002; Mouginot et al., 2012) which could have decreased the sublimation rate of the ice. Both of these factors, however, appear to have minimal effects on the general processes.

\subsubsection{Influence of obliquity}

Orbital spin-axis obliquity is a very important factor in the duration and the characteristics of the cold phase, because it controls the latitudinal distribution of the solar flux and thus the sublimation processes. We performed two simulations of the reference outflow channel event, at obliquities of $25^{\circ}$ and $65^{\circ}$, to complement the $45^{\circ}$ obliquity case presented initially. 

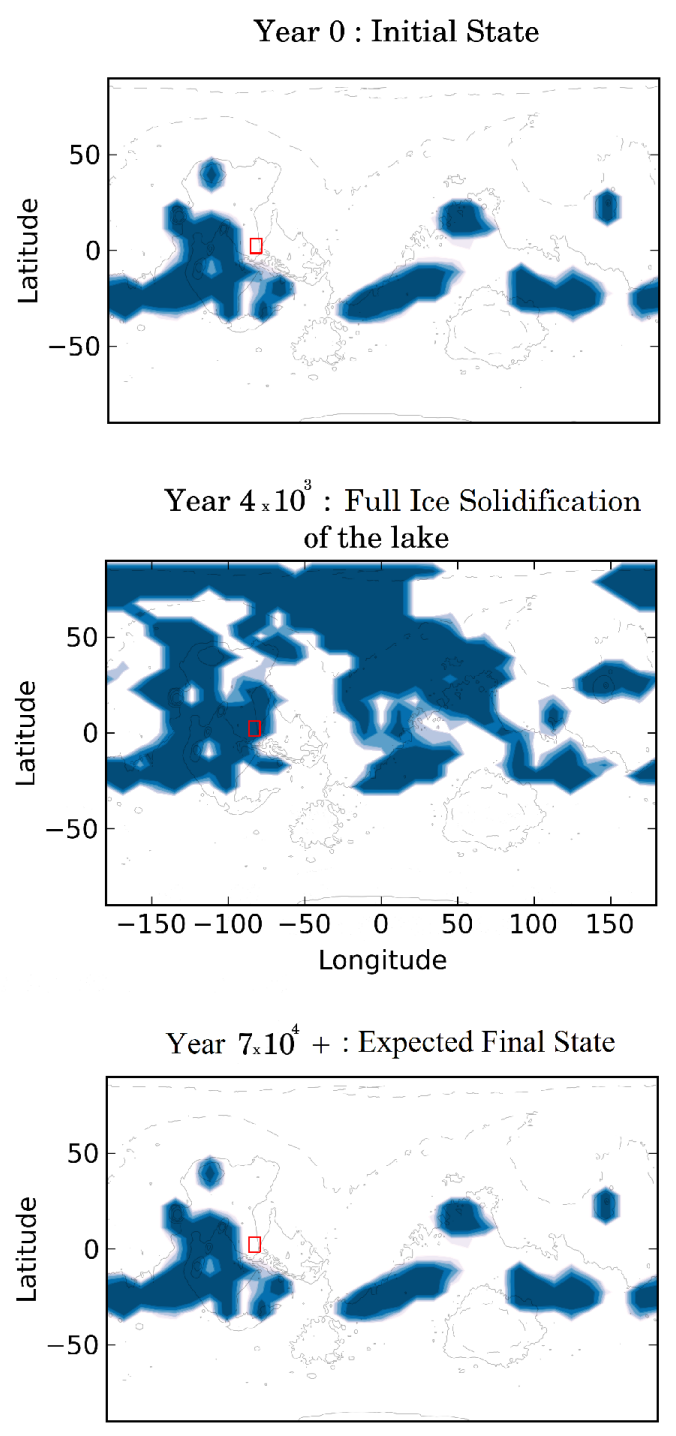

Year 1 : End of warm phase /

Full surface glaciation of the lake
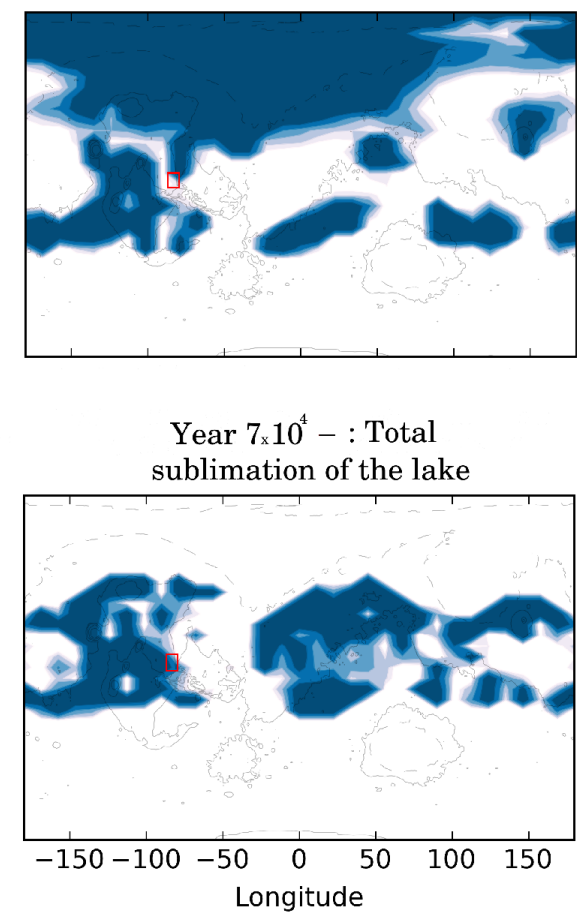

Year $7 \times 10^{4}-:$ Total sublimation of the lake

Snow deposit $(\mathrm{mm})$

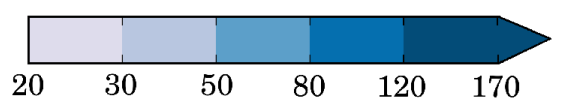

Figure 14: Ice deposit field after $0 / 1 / 4 \times 10^{3} / 7 \times 10^{4}$ martian years, corresponding to the main phases following the outflow channel reference event. The red rectangle corresponds to the West Echus Chasma Plateau area. 
In the low obliquity simulation, the sublimated ice migrates slowly toward the coldest points of the planet: the South pole and the North pole (in agreement with Wordsworth et al. (2013), Figure 4). The water present in the northern part of the lake is stable in the long term. In this situation, ice never accumulates in the region of West Echus Chasma.

In the high obliquity simulation, the water cycle is much more intense because the peak of insolation at high latitudes is higher. Approximately $\sim 55 \mathrm{~mm}$ of the sublimated northern lake ice migrates southward each year. The lifetime of the lake is thereby lowered to $\sim 9 \times 10^{3}$ martian years. For the same reasons as that in the reference simulation, a thick ice deposit is present in the region of West Echus Chasma Plateau. Yet, its duration, $\sim 10^{4}$ years, is almost 10 times less than in the reference simulation, more or less coincident with the lifetime of its supply (the frozen lake).

As a result, the lifetime of the ice located in West Echus Chasma area seems to be favored at obliquity $\sim 45^{\circ}$.

\section{The effect of surface pressure}

For many reasons (see discussion in section 2.2), the atmospheric pressure during the Late Hesperian epoch is not well constrained. We explore in this section the role of surface pressure on the climatic impact of outflow channels.

For this, we performed five different simulations of the same outflow channel event $\left(10^{6} \mathrm{~km}^{3}, 300 \mathrm{~K}\right.$ water released at $1 \mathrm{~km}^{3} \mathrm{~s}^{-1}$ in Echus Chasma) for five different surface pressures (40 mbar, 80 mbar, 0.2 bar (the reference simulation), 0.5 bar and 1 bar).

\subsection{Warm Phase}

Atmospheric pressure is one of the key factors that control the efficiency at which the warming of the atmosphere and the transport of water occur during the warm phase, as pointed out by Kite et al. (2011a).

1. The evaporation rate: Combining equations 9 and 15 for low amounts of water vapor, the evaporation rate $E$ can be written:

$$
E=\frac{C_{d} V_{1} P_{\mathrm{ref}} M_{\mathrm{CO}_{2}}}{R T_{1}} e^{\frac{L_{\mathrm{v}} M_{\mathrm{H}_{2} \mathrm{O}}}{R}\left(\frac{1}{T_{\mathrm{ref}}}-\frac{1}{T_{\text {surf }}}\right)} .
$$

Hence, the evaporation rate does not (directly) depend on the surface pressure and is mostly controlled by the temperature $\mathrm{T}_{\text {surf }}$ of the flow/lake. To first order (and 
this is confirmed by our simulations), the wind velocity $V_{1}$ and the atmospheric temperatures $T_{1}$ do not differ sufficiently from one atmospheric pressure to another to play a major role on the rate of evaporation.

2. The warming rate: The volumetric heat capacity of the atmosphere increases linearly with the volumetric mass density and thus the atmospheric pressure. For example, it takes approximately $\frac{1.0}{0.040}=25 \times$ more energy to warm a 1 bar atmosphere than a 40 mbar one.

When the outflow channel event occurs, the rate of warming of the atmosphere (in $\mathrm{K} / \mathrm{s}$ ) is roughly proportional to the evaporation rate (which is the main source of heating) and inversely proportional to the volumetric heat capacity of the atmosphere. In our simulations, it takes $\sim 10 / 40$ martian days - respectively for the $40 \mathrm{mbar} / 1 \mathrm{bar}$ case - for the atmospheric temperatures at $10 \mathrm{~km}$ to reach a plateau at $250 \mathrm{~K} / 220 \mathrm{~K}$, which correspond to a $+80 \mathrm{~K} /+30 \mathrm{~K}$ temperature increase (for initial temperatures equal to $170 \mathrm{~K} / 190 \mathrm{~K}$ ). This corresponds approximately to a factor of 10 in heating efficiency for these two endmember situations. The difference between the factor of 25 predicted and the factor of 10 obtained in our simulations is mostly due to two processes: advection and thermal emission to space.

The same two processes limit the growth of atmospheric temperatures. First, the advection tends to dilute the heat perturbation horizontally. In the 1 bar case, this is the dominant process for example. Second, the thermal emission to space acts as a very efficient negative feedback. This is, in fact, the first limiting process in the 40 mbar case.

The capability of an atmosphere to maintain high temperatures from the surface (where evaporation occurs) to the altitude where advection occurs is in fact the most important factor in the ability to transport water vapor globally and produce precipitation far from the region of evaporation. The warmer the atmospheric column above the lake is, the more water vapor will be possibly lifted and then transported globally by the high altitude winds.

Thin atmospheres (such as the 40 mbar) warm efficiently above the region of the flow, allowing the formation of a persistent water vapor plume that can transport (through advection) water vapor far from the flow/lake. In contrast, thick atmospheres (such as the 1 bar case) ironically do not manage to transport water efficiently because of the advection itself. The advection prevents the atmospheric temperatures above the lake from building up and thus the water vapor from accumulating. This limits the transport of water vapor and favors local precipitation. This is summarized by Figure 15 that shows the radial mean distribution (centered above the Northern Plains lake) of precipitation for the entire warm phase (first 500 days). Our experiments show that thin atmospheres are able to transport much 


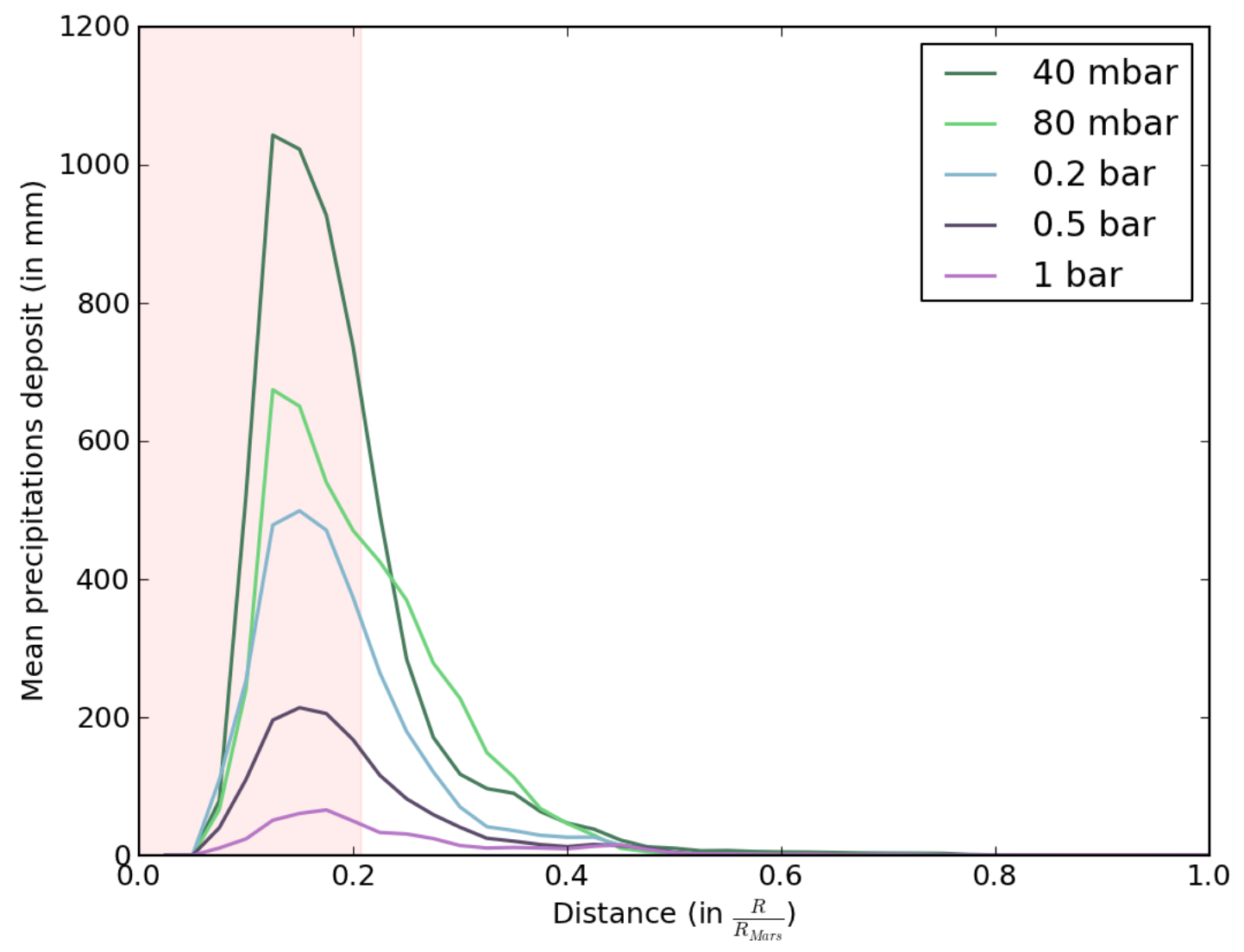

Figure 15: Radial precipitation distribution around the center of the lake $\left(70^{\circ} \mathrm{N},-30^{\circ} \mathrm{E}\right)$, averaged for the first 500 days following the outflow channel event, and for 5 different atmospheric pressures. The precipitation falling back on the lake/flow was removed from the plot. Because the lake is not circular, we used the pink color to represent the maximum radial extent of the lake. 
more water and for much longer distances than thick ones.

We compare in Figure 16 the spatial distribution of the precipitation (only snowfall, because rainfall occurs only above the lake) for the different atmospheric pressures. Whatever the surface pressure considered, the precipitation stays confined to the Northern Plains.

Another important aspect concerns the role of atmospheric pressure on the ability to melt the ice initially present / transported by the outflow event itself. Thin atmospheres, while able to reach temperatures in excess of $273 \mathrm{~K}$ above the flow, are not able to raise global temperatures significantly. First, the relaxation timescale of the temperature field is very low in such atmospheres because of the weak infrared absorption of the atmosphere. Second, outflow channel events under thin atmospheres generate a very large ice cover that reflects sunlight efficiently. As a result, an outflow channel of $10^{6} \mathrm{~km}^{3}$ that occurs under a 40 mbar atmosphere, leaves globally $\sim 1.5 \times 10^{4} \mathrm{~km}^{3}$ of water ice/snow (1.5\%) and is able to melt only $\sim 50 \mathrm{~km}^{3}(0.005 \%)$.

Thick atmospheres are initially warmer than thin atmospheres $(+30 \mathrm{~K}$ between the 1 bar and 40 mbar atmospheres). They also have a much more efficient infrared absorption and thus are better candidates to melt the deposited ice field. For example, an outflow channel of $10^{6} \mathrm{~km}^{3}$ that occurs under a 1 bar atmosphere, leaves globally $\sim 4 \times 10^{3} \mathrm{~km}^{3}$ of water ice/snow $(0.4 \%)$ and is able to melt $\sim 110 \mathrm{~km}^{3}(0.011 \%)$.

Nonetheless, this melting occurs only in the Northern Plains, in the close vicinity of the lake, because such thick atmospheres do not transport much ice anywhere on the planet in any case. In addition, ice albedo feedback (which is yet lower for thicker atmospheres) and the high volumetric heat capacity (lower heat perturbation) of such atmospheres contribute to lower the possibility of reaching melting temperatures.

Whatever the value of the surface pressure, the ability of the atmosphere to produce liquid water from melting is very limited.

\subsection{Cold Phase}

The water cycle during the cold phase is, in contrast, more intense for thick atmospheres than for thin ones. The sublimations rates are higher because global temperatures (and also summer temperatures) are also higher. At the end of the warm phase, the mean global temperatures for the $40 \mathrm{mbar} / 1$ bar simulations are respectively $\sim 193 \mathrm{~K}(3.5 \mathrm{~K}$ lower than the control simulation) and $\sim 226 \mathrm{~K}(1 \mathrm{~K}$ 


\section{Ice Layer Deposit}
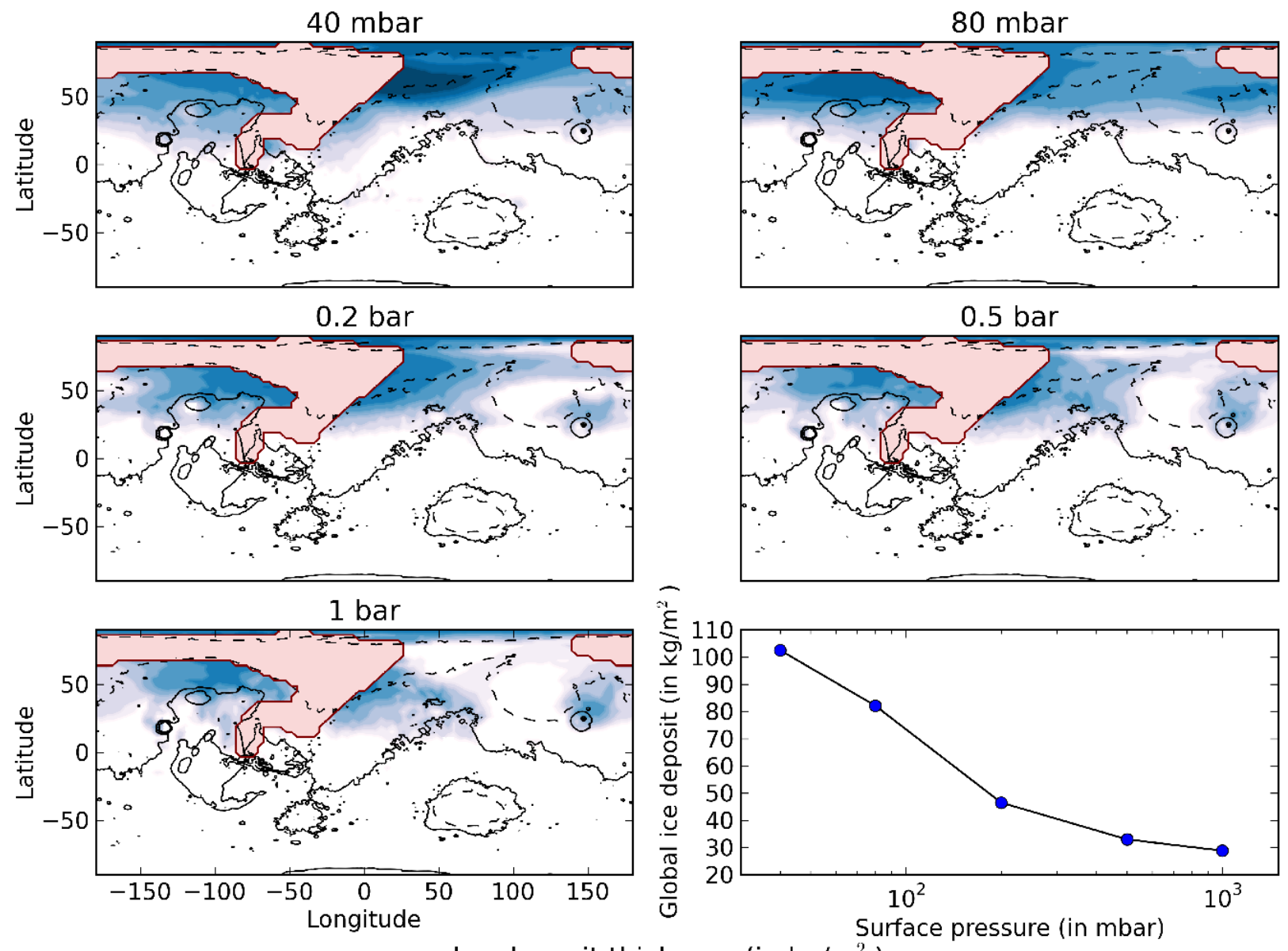

Ice deposit thickness (in $\mathrm{kg} / \mathrm{m}^{2}$ )

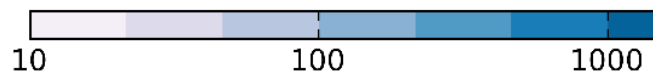

Figure 16: Final Ice layer deposit map (in $\mathrm{kg} \mathrm{m}^{-2}$ ) after 1 martian year of simulations, for five different surface pressures ( 40 mbar, 80 mbar, 0.2 bar, 0.5 bar and 1 bar). The pink color denotes the regions where the flow passed through on the way to the lake. 


\section{Maximum surface liquid water in the year following the event}

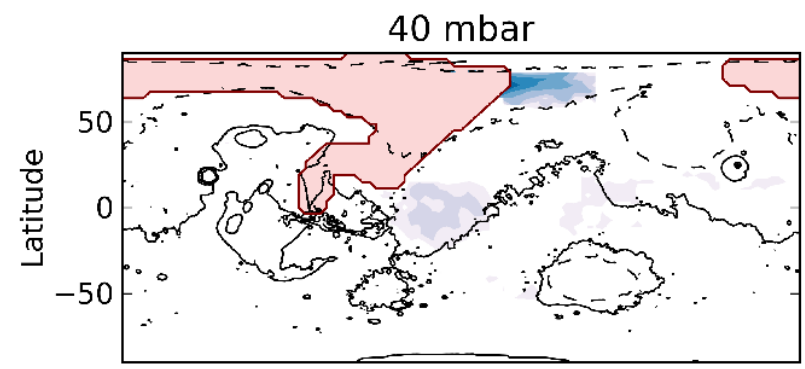

\section{2 bar}

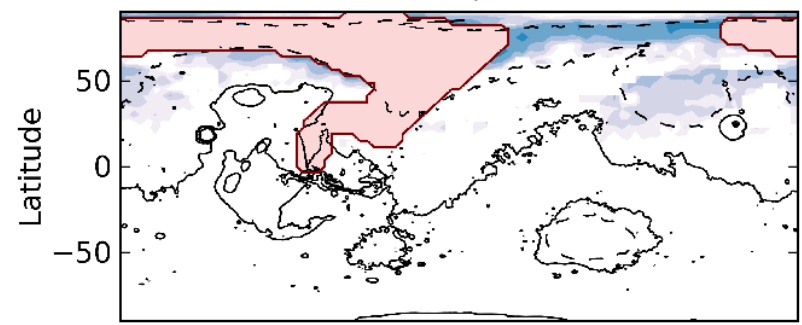

\section{1 bar}

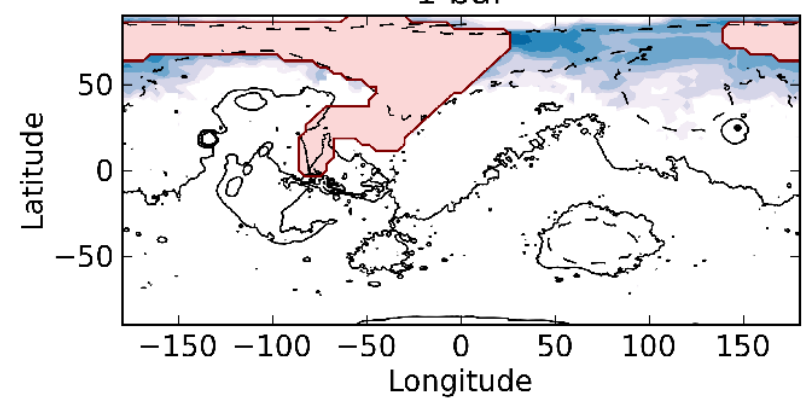

80 mbar

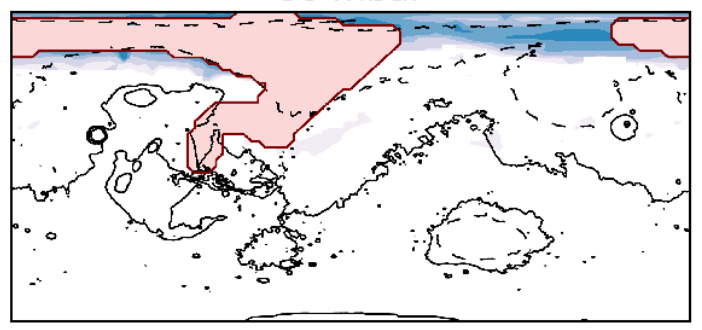

0.5 bar
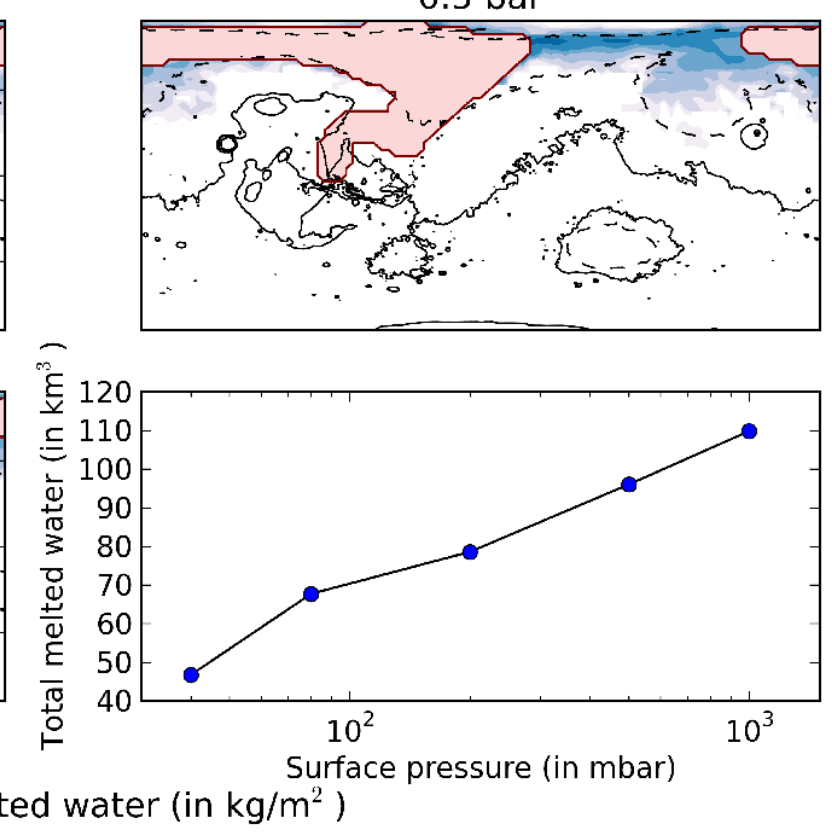

Maximum melted water (in $\mathrm{kg} / \mathrm{m}^{2}$ )

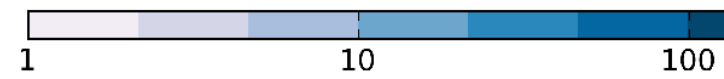

Figure 17: Maximum surface liquid water after 1 year of simulation and for five different surface pressures (40 mbar, 80 mbar, 0.2 bar, 0.5 bar and 1 bar). The pink color denotes the regions where the flow passed through on the way to the lake. 
lower than the control simulation). This difference is due to the increased ice cover following the outflow event.

In the 1 bar simulation (thick case), the lifetime of the frozen lake is $\sim 5 \times 10^{4}$ martian years, slightly lower than in the reference simulation. The climatic response during the cold phase behaves more or less in the same manner as in the reference 0.2 bar simulation.

In the 40 mbar simulation (thin case) however, because the water cycle is too weak (sublimation rate of the lake of $2 \mathrm{~mm} /$ year; lifetime of the frozen lake $\sim 2 \times 10^{5}$ years), the southward flux of the atmospheric water ice is not high enough to allow the presence of stable ice in the area of the West Echus Chasma Plateau.

More generally, atmospheres with pressure higher than 80 mbar seem necessary to produce ice deposits in the region of West Echus Chasma Plateau.

\section{Extreme parameterizations}

In this section, we study several scenarios that may deeply affect the climatic impact of outflow events: 1 . the intensity of the event and 2. the effect of clouds and precipitation.

\subsection{Intensity of the event}

Because outflow channel events such as the one presented in Section 4 fail to produce rainfall/transient warming, it is tempting to explore even more extreme parameterizations of the outflow events.

\subsubsection{Temperature of the flow}

The temperature of the groundwater released during outflow events is not well constrained (see section 2.1.1). Hence, we used the temperature of the flow as a tuning parameter to explore the sensitivity of our results to the intensity of the outflow event. We performed three simulations of the same outflow event $\left(10^{6} \mathrm{~km}^{3}\right.$, released in Echus Chasma) for three different groundwater temperatures: $280 \mathrm{~K}, 300 \mathrm{~K}$ (reference simulation) and $320 \mathrm{~K}$.

As expected, the warmer the water, the more intense the climatic effect becomes. For example, at the peak of the warm phase, the $320 \mathrm{~K}$ event is able to carry approximately $8 \times$ more water vapor than in the reference simulation because 
atmospheric warming processes are amplified by the temperature (evaporation/condensation cycle, IR emission of the flow, ...). Consequently, $25 \%$ of the precipitation following the $320 \mathrm{~K}$ event is rainfall (respectively $10 \% / 0 \%$ for the reference/280 K simulations). Yet, rainfall still occurs exclusively above the lake $(70 \%)$ or in the northern lowlands of Mars $(30 \%)$. Snow precipitation also remains confined to the Northern Plains down to $15^{\circ} \mathrm{N}\left(25 / 40{ }^{\circ} \mathrm{N}\right.$ for the $300 \mathrm{~K} / 280 \mathrm{~K}$ simulations).

The amount of water ice transported (Figure 18) and melted (Figure 19) after outflow channel events with $280 \mathrm{~K} / 300 \mathrm{~K} / 320 \mathrm{~K}$ water shows that in all cases, the mechanism of advection/cooling to space is very efficient, and as a result, the duration of the warm phase is approximately the same ( $\sim 500$ days $)$ between the reference and the $320 \mathrm{~K}$ simulations.

We note that, at the end of the warm phase, because the amount of ice transported (and the area of the deposit with it) increases with the initial temperature of the flow, the average surface albedo raises and the mean temperatures decrease: Warmer flows lead to colder states.

\subsubsection{Magnitude of the event: from small outflows to oceans.}

Recent work (Andrews-Hanna and Phillips, 2007; Harrison and Grimm, 2008) has suggested that outflow channels were preferentially carved by multiple events of reduced sizes $\left(\sim 10^{3} \mathrm{~km}^{3}\right)$ rather than by large $\left(>10^{5} \mathrm{~km}^{3}\right)$ single outflows. We performed simulations for different volumes of water at $300 \mathrm{~K}$ and released in Echus Chasma at a rate of $1 \mathrm{~km}^{3} \mathrm{~s}^{-1}$, from $10^{3} \mathrm{~km}^{3}$ (consistent with the most recent estimations of outflow volumes) to $10^{7} \mathrm{~km}^{3}$ (ocean case). Figure 20 shows the final position of the lake as a function of the initial volume of water. The $10^{6} \mathrm{~km}^{3}$ case is the reference simulation.

Our results show that the large outflows, during the warm phase, transport much more water than the small ones (cumulative). Small outflows (typically $\sim 10^{3}-10^{4} \mathrm{~km}^{3}$ ) have a small wetted area (typically $0.15-0.41 \times 10^{6} \mathrm{~km}^{3}$ ) and a small initial heat reservoir, so that they cannot warm the atmospheric column above the flow/lake sufficiently to transport water vapor into the neighbouring regions. Small outflow events inject more or less the same amount of water vapor (in proportion) than large ones, but they are not able to transport it far from the flow/lake. For example, $2 \times 10^{2}$ events of $5 \times 10^{3} \mathrm{~km}^{3}$ transport 2 orders of magnitude less ice outside the flow/lake than a large $10^{6} \mathrm{~km}^{3}$ one (reference simulation). Moreover, large outflows are able to generate precipitation up to $5000 \mathrm{~km}$ from the edge of the flow/lake whereas small ones cannot produce any precipitation at a distance greater than $\sim 400 \mathrm{~km}$ (typically the size of 2 GCM 


\section{Ice Layer Deposit at the end of the warm phase}
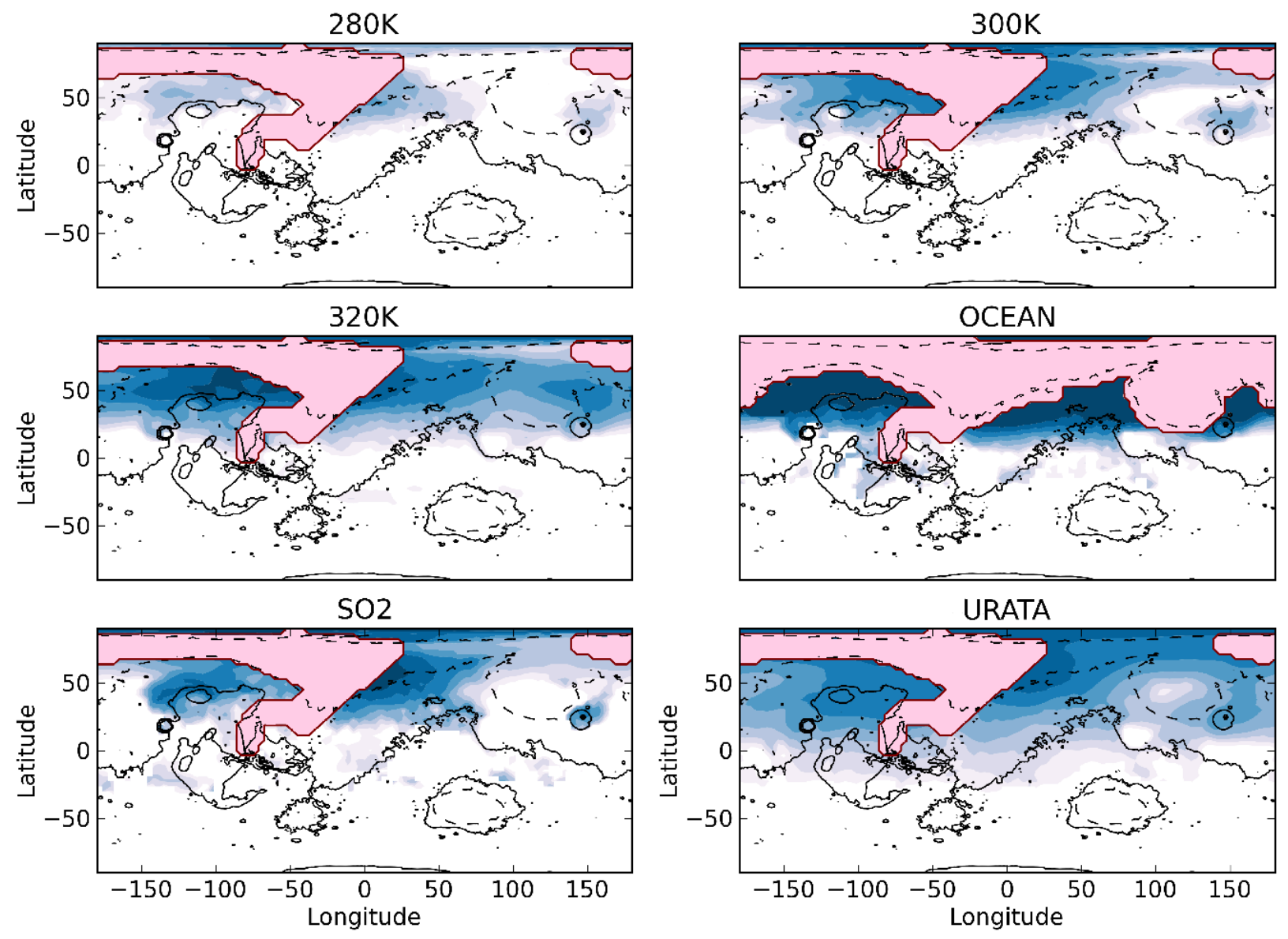

Ice deposit thickness (in $\mathrm{kg} / \mathrm{m}^{2}$ )

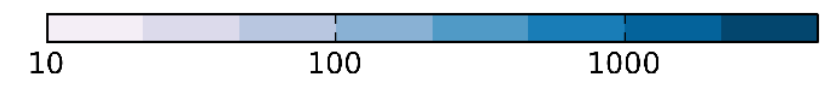

Figure 18: Final Ice layer deposit map (in $\mathrm{kg} \mathrm{m}^{-2}$ ) after 1 martian year of simulations (4 martian years for the ocean case), for six different simulations: 1. $280 \mathrm{~K}$ outflow, 2. $300 \mathrm{~K}$ outflow (reference case), 3. $320 \mathrm{~K}$ outflow, $4.10^{7} \mathrm{~km}^{3}$ ocean case, $5.1 \% \mathrm{SO}_{2}$ case and 6. $1_{0}=\infty$ (no precipitation case). The pink color denotes the regions where the flow passed through on the way to the lake. 


\section{Maximum surface liquid water in the year following the event}
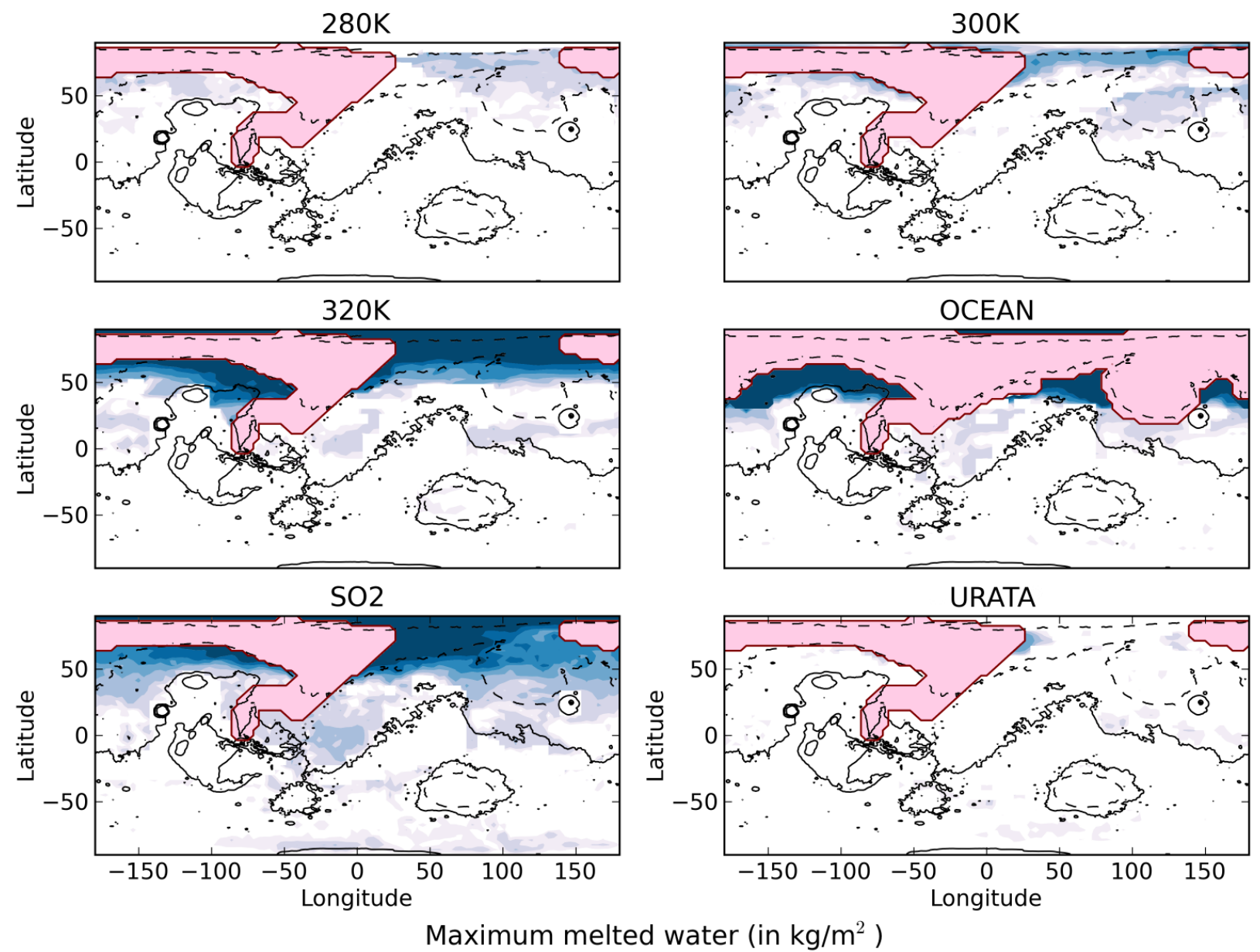

Maximum melted water (in $\mathrm{kg} / \mathrm{m}^{2}$ )

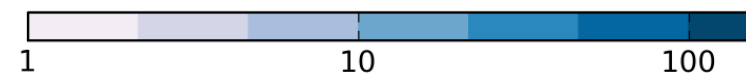

Figure 19: Maximum surface melted liquid water after 1 year of simulation (4 martian years for the ocean case) and for 6 different simulations: $1.280 \mathrm{~K}$ outflow, 2. $300 \mathrm{~K}$ outflow (reference case), 3. $320 \mathrm{~K}$ outflow, $4.10^{7} \mathrm{~km}^{3}$ ocean case, 5 . $1 \% \mathrm{SO}_{2}$ case and $6.1_{0}=\infty$ (no precipitation case). The pink color denotes the regions where the flow passed through on the way to the lake. 


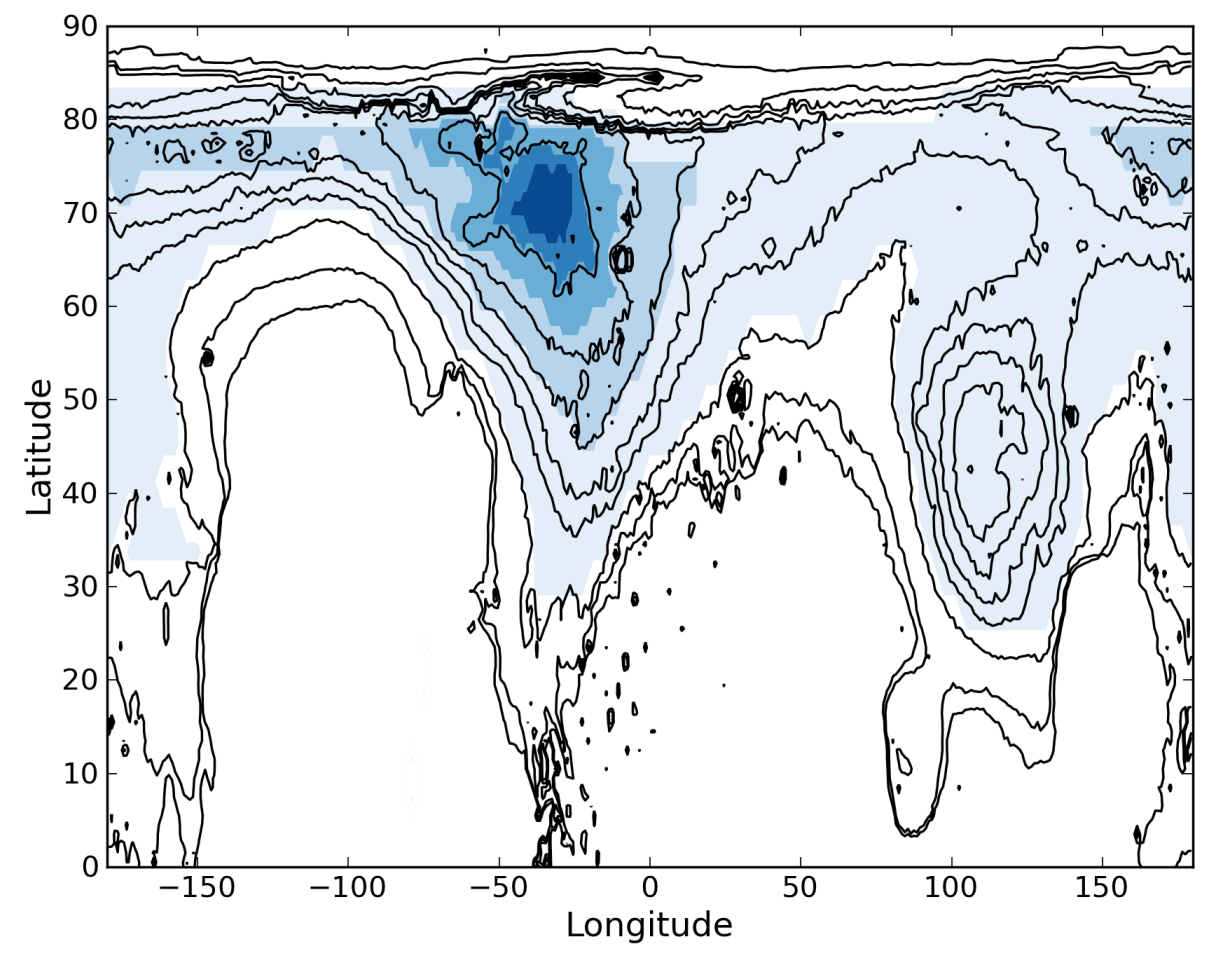

Figure 20: Stable water lake size obtained in our simulations depending on the volume of water released. Contour fills are for volumes of $10^{3}, 10^{4}, 10^{5}, 10^{6}$ (reference case) and $10^{7} \mathrm{~km}^{3}$ (ocean case). These correspond respectively to wetted areas of $0.15,0.41,1.19,4.15$ and 20.4 millions of $\mathrm{km}^{2}$. 
grids).

We did not explore in detail the effect of the discharge rate, which has a net impact on the size and duration of the wetted area (and thus on the evaporation and the albedo), but also on the intensity of the event. Nonetheless, the climatic response to lower discharge rate events $\left(<10^{9} \mathrm{~m}^{3} \mathrm{~s}^{-1}\right)$ was found to be lower, because in such cases the temperatures and the amount of water vapor struggle to build up above the flow/lake.

Because large outflows seem to be much better candidates for generating precipitation globally, we examined the extreme case of a catastrophic outflow event of $10^{7} \mathrm{~km}^{3}$ released simultaneously by all of the circum-Chryse outflow channels (Kasei, Ares, Tiu, Simu Vallis, etc.). This possibility, sometimes called the MEGAOUTFLO (Mars Episodic Glacial Atmospheric Oceanic Upwelling by Thermotectonic Flood Outburst) hypothesis (Baker et al., 1999), speculates that such events could warm Mars during periods of $10^{4}-10^{5}$ years through a transient greenhouse effect provoked in part by the injection of large amounts of water vapor.

Our experiments show that such events cannot sustain long-term greenhouse effects, whatever the size and the temperatures considered for the northern lake/see/ocean. After 3.5 martian years, for the outflow event described above, the surface of the lake/see/ocean becomes totally frozen. The thermal infrared emission to space (enhanced by the heat horizontal advection and by the water vapor advection that release latent heat because of adiabatic cooling; see Figure 21 for the detailed mechanism) acts very efficiently to cool the planet. The ice deposited on the Northern Plains slopes (Figure 18) also enhances the cooling through a depletion of surface solar absorption. As a result, in such a scenario, rainfall/snowmelt still only occurs in the lowest northern lowlands (see Figure 19) of the planet (far from the region of interests).

In summary, the most intense outflow channel events possible are not able to sustain a global greenhouse warming. Such events only manage to warm up the atmosphere regionally, in the Northern Plains, and only for a few years at best. Consequently, rainfall (and snowmelt) occur only in the neighbourhood regions of the final stable lake. After complete surface freezing of the lake, the climate becomes much colder than initially (due to the increase of the surface albedo), making the snowmelt even more difficult.

We note that we did not take into account the modification of the topography by the presence of a lake/see/ocean, which might be a concern for very high volumes of water $\left(\geq 10^{7} \mathrm{~km}^{3}\right)$. It could reduce significantly the role of adiabatic cooling and thus favor the transport/deposit of water further south. 

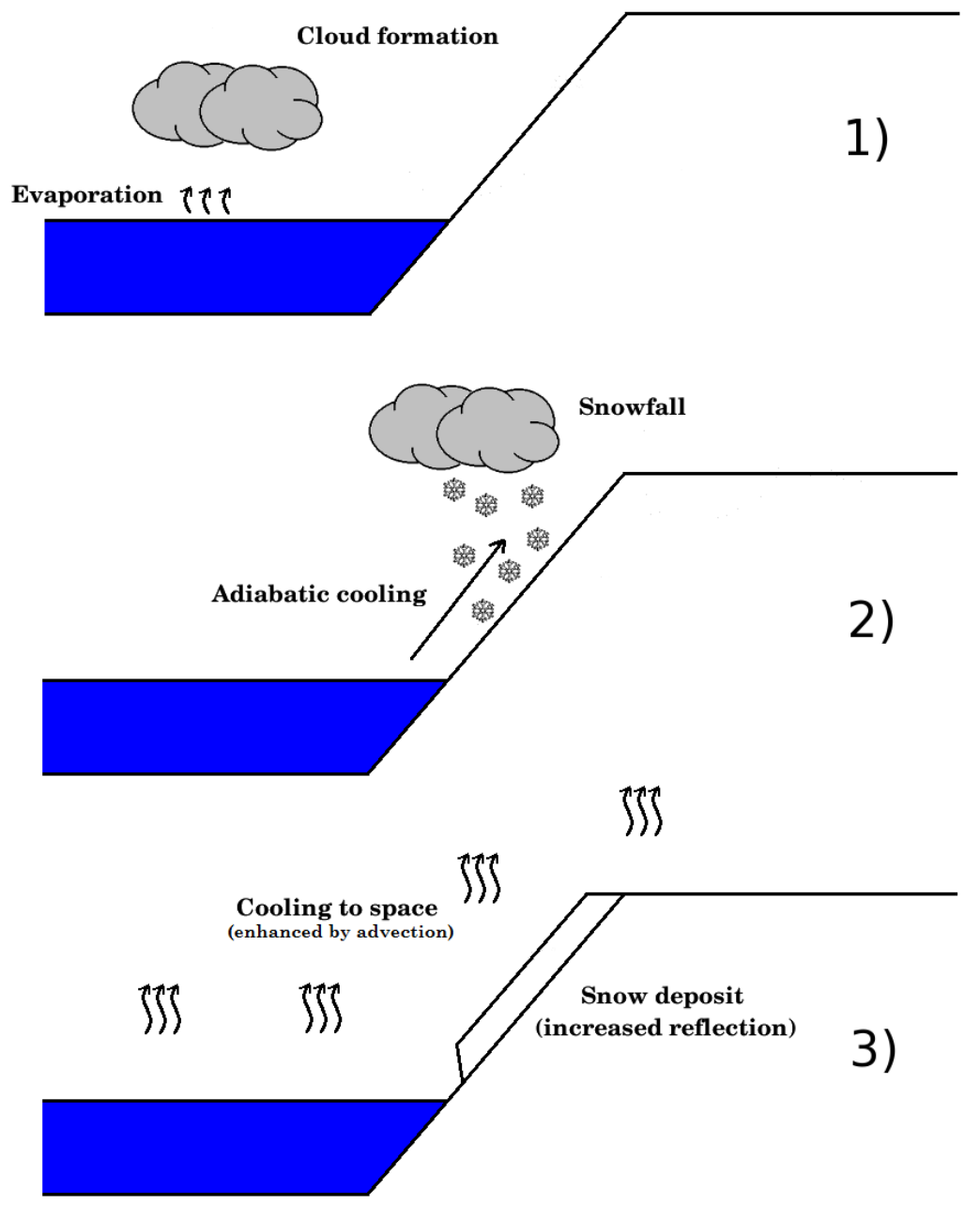

Figure 21: Why is a martian Northern ocean short-lived and unable to induce rain? The water vapor and the clouds that build up above the ocean (1) are advected southward (2). Because of adiabatic cooling, snowfall occurs from the edges of the ocean (starting from $70^{\circ} \mathrm{N}$, see Figure 20) to the highest parts of the planet (the 'Icy Highlands'). The advection of heat (increasing the surface of thermal infrared emission) and the increase of reflection (because of the snow deposit) both contribute to an intense cooling of the ocean and to reduced precipitation. 


\section{Discussion}

\subsection{Role of the atmospheric composition.}

In this analysis, we made the assumption that the Late Hesperian martian atmosphere was made of $100 \% \mathrm{CO}_{2}$ (and some water vapor). Outflow channel events under a $\mathrm{CO}_{2}$ dominated atmosphere seem not to be able to provoke long-term warming or precipitation at the global scale.

Ouflow channel formation events are very likely related to intense volcanic episodes during martian history (Baker et al., 1991; Head et al., 2002). During these periods, it is believed that volcanic gases like $\mathrm{SO}_{2}$ may have been massively released [see section 1. of Kerber et al. (2015) for more details].

We performed a simulation of an outflow channel event under the same conditions as in section 4 , but this time with $1 \%$ of $\mathrm{SO}_{2}$. Figures 18 and 19 show the corresponding amount of water ice transported/melted after the event. Small amounts of $\mathrm{SO}_{2}$ (2 mbar here) are sufficient to raise the global atmospheric temperatures by several tens of Kelvins and thus to favor the transport of water vapor/water ice globally and create precipitation far from the Northern Plains stable lake.

However, using the same GCM, Kerber et al. (2015) (and earlier, Tian et al. (2010) ) have shown that massive volcanic $\mathrm{SO}_{2}$ outgassing cannot lead to a global and substantial warming, because sulfur aerosols that would form at the same time have a very strong cooling effect, even in small amounts.

We also believe that, under more realistic parameterizations that would take into account sulfur aerosols (e.g. Halevy and Head (2014)), the outflow channel climatic impact would be also very limited.

\subsection{The role of clouds and precipitation.}

The radiative effect of clouds is one of the main sources of uncertainty in GCMs and thus also on the consistency of our results.

In particular, it has been suggested (Urata and Toon, 2013) that high altitude ('cirrus-like') water ice clouds may trigger warm climates on Mars even under a faint young sun. This scenario requires four assumptions: 1) Water ice particles that have sizes $>10$ microns; 2 ) that the rate of precipitation is very low (in order to extend the lifetime of the clouds); 3) When present, clouds need to completely cover a grid cell (no partial cloud cover); 4) Lastly, it also requires an initial 'warm' state, for example an outflow channel event. 
To explore in a basic manner the role of clouds and precipitation on the climatic impact of outflow channels, we performed a simulation of the reference outflow channel event in which we eliminated the precipitation resulting from coalescence $\left(1_{0}=\infty\right)$. For this case, the vertical motion of the ice particles is governed only by gravitational sedimentation. Figure 11 shows that the total cloud cover is near $100 \%$ over all the planet during the first year following the event, because of the intense evaporation coupled with the increased lifetime of clouds.

We found that neglecting coalescence and the subsequent precipitation led to ice deposits that extend much more areally than in the reference case (Figure 18), because the lifetime of ice particles increases substantially. In such a situation, the global cloud cover (during the year following the event) has a net positive radiative impact on the global energy balance of $+12 \mathrm{~W} \mathrm{~m}^{-2}\left(+21.3 \mathrm{~W} \mathrm{~m}^{-2}\right.$ of IR warming; - 9.2 $\mathrm{W} \mathrm{m}^{-2}$ of solar absorption). This is $\sim+11 \mathrm{~W} \mathrm{~m}^{-2}$ higher than in the reference simulation.

However, because the ice field produced by the event extends to a much larger area, the global albedo increases and contributes approximately $6 \mathrm{~W} \mathrm{~m}^{-2}$ of cooling. Moreover, because of advection processes, this also increases the horizontal extent of the heat perturbation and thereby the global infrared emission to space. Under clear sky conditions, this would lead to an extra cooling of $\sim 5 \mathrm{~W} \mathrm{~m}^{-2}$ compared to the reference simulation.

As a consequence, the total rate of cooling is more or less the same $\left(\sim 15 \mathrm{~W} \mathrm{~m}^{-2}\right)$ as that in the reference simulation $\left(1_{0}=0.001\right)$. The duration of the warm phase is also more or less the same than in the reference simulation ( $\sim 500$ days).

We also note that the seasonal melting of the deposited ice (see Figure 19) would be very limited in such scenarios, because of the increased solar reflection by the clouds. In addition, because the ice field produced by the event extends over a large region (Figure 18), the planet becomes much colder one year after the event than initially.

Nonetheless, we highly encourage further studies to explore in more detail the possibility of warming early Mars through water ice clouds (as recently done by Ramirez and Kasting (2017)).

\subsection{Conclusions}

In this analysis, we explored the climatic impact of a wide range of outflow channel events under many possible conditions.

We find that even considering outflow events with intensity (in volumes and temperatures of water released) that exceed by far the most recent estimates, the 
short term climatic response is still very limited. The duration of the 'warm' phase that follows the outflow events is completely controlled by the total depth and temperature of the lake that is formed and is, in practice, no more than few years for the most extreme cases $\left(10^{7} \mathrm{~km}^{3}\right.$ of water warmed at $300 \mathrm{~K}$, e.g. ocean case). In other words, outflow events fail to trigger greenhouse-sustained warm episodes. Moreover, the precipitation (almost exclusively snowfall) produced by the events during their warm phase is limited and confined to the Northern Plains, in the area neighbouring the water outflow. These results are robust over a wide range of atmospheric pressures and external conditions (e.g. obliquity and season).

We also find that the intensity of outflow channel event effects can be significantly influenced by the atmospheric pressure which is not well constrained for the Hesperian era. Thin atmospheres $(\mathrm{P}<80 \mathrm{mbar})$, because of their low volumetric heat capacity, can be warmed efficiently. This can trigger the formation of a convective plume, a very efficient mechanism to transport water vapor and ice to the global scale. Thick atmospheres $(\mathrm{P}>0.5$ bar) have difficulty in producing precipitation far from the outflow water locations but they are more suited to generate snowmelt. Nonetheless, outflow channel formation events are unable, whatever the atmospheric pressure, to produce rainfall or significant snowmelt at latitudes below $40^{\circ} \mathrm{N}$.

During the 'cold phase' that follows the solidification to ice of the outflow water, the body of water ice emplaced in the Northern Plains has a major contribution to the water cycle. The ice is sublimated seasonally and transported progressively southward toward the 'Icy Highlands' regions by the processus of adiabatic cooling. We find that under favorable conditions (obliquity $\sim 45^{\circ}$, atmospheric pressure $\geqslant$ 80 mbar), ice deposits can be stabilized in the West Echus Chasma Plateau area. For an initial $10^{6} \mathrm{~km}^{3}$ body of water ( 0.2 bar atmospheric pressure, $45^{\circ}$ obliquity), they can be present during $10^{5}$ martian years. However, seasonal melting related to solar forcing seems difficult because 1) the West Echus Chasma Plateau is not ideally located, and 2) the presence of (high albedo) snow at the surface has a significant cooling effect. The global temperatures after outflow events can thus easily be lowered by few Kelvins making the solar melting possibility even more difficult. Therefore, in this scenario, localized warming such as geothermal activity or meteoritic impacts would be required to explain the formation of valley networks dated to the Late Hesperian era and yet observed at this specific location. 


\section{References}

Andrews-Hanna, J. C. and Phillips, R. J. (2007). Hydrological modeling of outflow channels and chaos regions on Mars. Journal of Geophysical Research, 112.

Bahcall, J. N., Pinsonneault, M. H., and Basu, S. (2001). Solar models: Current epoch and time dependences, neutrinos, and helioseismological properties. The Astrophysical Journal, 555:990-1012.

Baker, V., Strom, R., Doh, J., Gulick, V., Kargel, J., Komatsu, G., Ori, G., and Rice, J. (1999). Mars - oceanus borealis, ancient glaciers, and the megaoutflo hypothesis.

Baker, V. R. (1982). The channels of Mars. University of Texas Press, Austin.

Baker, V. R. (2001). Water and the martian landscape. Nature, pages 228-236.

Baker, V. R., Carr, M. H., Gulick, V. C., Williams, C. R., and Marley, M. S. (1992). Channels and valley networks, pages 493-522. University of Arizona Press, Tucson.

Baker, V. R., Strom, R. G., Gulick, V. C., Kargel, J. S., Komatsu, G., and Kale, V. S. (1991). Ancient Oceans, ice sheets and the hydrological cycle on Mars. Nature, 352.

Baranov, Y. I., Lafferty, W. J., and Fraser, G. T. (2004). Infrared spectrum of the continuum and dimer absorption in the vicinity of the $\mathrm{O}_{2}$ vibrational fundamental in $\mathrm{O}_{2} / \mathrm{CO}_{2}$ mixtures. Journal of Molecular Spectroscopy, 228:432-440.

Bathurst, J. C. (1993). Flow resistance through the channel network. Channel Network Hydrology, pages 69-98.

Bibring, J.-P., Langevin, Y., Mustard, J. F., Poulet, F., Arvidson, R., Gendrin, A., Gondet, B., Mangold, N., Pinet, P., and Forget, F. (2006). Global Mineralogical and Aqueous Mars History Derived from OMEGA/Mars Express Data. Science, 312:400-404.

Bolmont, E., Libert, A.-S., Leconte, J., and Selsis, F. (2016). Habitability of planets on eccentric orbits: Limits of the mean flux approximation. Astronomy and Astrophysics, 591:A106. 
Carr, M. H. (1996). Water on Mars. New York: Oxford University Press.

Carter, J., Poulet, F., Bibring, J.-P., Mangold, N., and Murchie, S. (2013). Hydrous minerals on Mars as seen by the CRISM and OMEGA imaging spectrometers: Updated global view. Journal of Geophysical Research (Planets), 118:831-858.

Cassanelli, J. P., Head, J. W., and Fastook, J. L. (2015). Sources of water for the outflow channels on Mars: Implications of the Late Noachian "icy highlands" model for melting and groundwater recharge on the Tharsis rise. Planet. Space Sci., 108:54-65.

Charnay, B., Forget, F., Wordsworth, R., Leconte, J., Millour, E., Codron, F., and Spiga, A. (2013). Exploring the faint young sun problem and the possible climates of the archean earth with a 3-d gcm. Journal of Geophysical Research : Atmospheres, 118:414-431.

Chevrier, V., Poulet, F., and J.-P., B. (2007). Early geochemical environment of mars as determined from thermodynamics of phyllosilicates. 448:6063.

Clifford, S. M. (1993). A model for the hydrologic and climatic behavior of water on mars. J. Geophys. Res., 98:10,973-11,016.

Clifford, S. M. and Parker, T. (2001). The evolution of the martian hydrosphere: Implications for the fate of a primordial ocean and the current state of the northern plains. Icarus, 154:40-79.

Clough, S., Kneizys, F., and Davies, R. (1989). Line shape and the water vapor continuum. Atmospheric Research.

Codron, F. (2012). Ekman heat transport for slab oceans. Climate Dynamics, 38:379-389.

Craddock, R. A. and Howard, A. D. (2002). The case for rainfall on a warm, wet early Mars. Journal of Geophysical Research (Planets), 107:21-1.

Craddock, R. A. and Maxwell, T. A. (1993). Geomorphic evolution of the Martian highlands through ancient fluvial processes. J. Geophys. Res., 98:3453-3468.

Ehlmann, B. L., Mustard, J. F., Murchie, S. L., Bibring, J.-P., Meunier, A., Fraeman, A. A., and Langevin, Y. (2011). Subsurface water and clay mineral formation during the early history of Mars. Nature, 479:53-60. 
Emanuel, K. A. and Ivkovi-Rothman, M. (1999). Development and Evaluation of a Convection Scheme for Use in Climate Models. Journal of Atmospheric Sciences, 56:1766-1782.

Fassett, C. I. and Head, J. W. (2008). The timing of martian valley network activity: Constraints from buffered crater counting. Icarus, 195:61-89.

Fastook, J. L. and Head, J. W. (2014). Amazonian mid- to high-latitude glaciation on Mars: Supply-limited ice sources, ice accumulation patterns, and concentric crater fill glacial flow and ice sequestration. Planetary and Space Science, 91:60-76.

Fastook, J. L. and Head, J. W. (2015). Glaciation in the Late Noachian Icy Highlands: Ice accumulation, distribution, flow rates, basal melting, and top-down melting rates and patterns. Planetary and Space Science, 106:82-98.

Fastook, J. L., Head, J. W., Marchant, D. R., Forget, F., and Madeleine, J.-B. (2012). Early Mars climate near the Noachian-Hesperian boundary: Independent evidence for cold conditions from basal melting of the south polar ice sheet (Dorsa Argentea Formation) and implications for valley network formation. Icarus, 219:25-40.

Forget, F., Hourdin, F., and Talagrand, O. (1998). $\mathrm{CO}_{2}$ snow fall on Mars: Simulation with a general circulation model. Icarus, 131:302-316.

Forget, F. and Leconte, J. (2014). Possible climates on terrestrial exoplanets. Phil. Trans. R. Soc.

Forget, F., Vangvichith, M., and Bertrand, T. (2014). What will pluto's atmosphere look like ? predictions from a global climate model including the methane cycle. In AGU Fall Meeting Abstracts.

Forget, F., Wordsworth, R., Millour, E., Madeleine, J.-B., Kerber, L., Leconte, J., Marcq, E., and Haberle, R. M. (2013). 3D modelling of the early martian climate under a denser $\mathrm{CO}_{2}$ atmosphere: Temperatures and $\mathrm{CO}_{2}$ ice clouds. Icarus, 222:81-99.

Fu, Q. and Liou, K. N. (1992). On the correlated k-distribution method for radiative transfer in nonhomogeneous atmospheres. Journal of the Atmospheric Sciences, 49. 
Galperin, B. A., Kantha, L. H., Hassid, S., and Rosati, A. (1988). A quasi-equilibrium turbulent energy model for geophysical flows. J. Atmos. Sci., 45:55-62.

Golombek, M. P., Grant, J. A., Crumpler, L., Greeley, R., Arvidson, R., Bell, J. F., Weitz, C. M., Sullivan, R., Christensen, P. R., Soderblom, L. A., and Squyres, S. W. (2006). Erosion rates at the Mars Exploration Rover landing sites and long-term climate change on Mars. Journal of Geophysical Research, 111.

Gough, D. O. (1981). Solar interior structure and luminosity variations. Solar Phys., 74:21-34.

Grott, M., Morschhauser, A., Breuer, D., and Hauber, E. (2011). Volcanic outgassing of $\mathrm{CO}_{2}$ and $\mathrm{H}_{2} \mathrm{O}$ on Mars. Earth and Planetary Science Letters, 308:391-400.

Gruszka, M. and Borysow, A. (1998). Computer simulation of the far infrared collision induced absorption spectra of gaseous CO2. Molecular Physics, 93:1007-1016.

Guerlet, S., Spiga, A., Sylvestre, M., Indurain, M., Fouchet, T., Leconte, J., Millour, E., Wordsworth, R., Capderou, M., Bezard, B., and Forget, F. (2014). Global climate modeling of Saturn's atmosphere. Part I: Evaluation of the radiative transfer model. Icarus, 238:110-124.

Gulick, V. (2001). Origin of the valley networks on Mars: a hydrological perspective. Geomorphology, 37:241-268.

Gulick, V. C. (1998). Magmatic intrusions and a hydrothermal origin for fluvial valleys on Mars. Journal of Geophysical Research, 103:19365-19388.

Gulick, V. C. and Baker, V. R. (1989). Fluvial valleys and Martian palaeoclimates. Nature, 341:514-516.

Gulick, V. C. and Baker, V. R. (1990). Origin and evolution of valleys on Martian volcanoes. Journal of Geophysical Research, 95:14325-14344.

Gulick, V. C., Tyler, D., McKay, C. P., Kargel, J. S., and Haberle, R. M. (1997). Episodic Ocean-Induced $\mathrm{CO} 2$ Greenhouse on Mars: Implications for Fluvial Valley Formation. Icarus, 130. 
Halevy, I. and Head, III, J. W. (2014). Episodic warming of early Mars by punctuated volcanism. Nature Geoscience, 7:865-868.

Hansen, J. E. and Travis, L. D. (1974). Light scaterring in planetary atmosphere. Space Sci. Rev., 16:527-610.

Harrison, K. P. and Grimm, R. E. (2005). Groundwatercontrolled valley networks and the decline of surface runoff on early Mars. Journal of Geophysical Research, 110.

Harrison, K. P. and Grimm, R. E. (2008). Multiple flooding events in Martian outflow channels. Journal of Geophysical Research, 113.

Hartmann, W. K. and Neukum, G. (2001). Cratering Chronology and the Evolution of Mars. Space Science Reviews, 96:165-194.

Head, J. W., Kreslavsky, M. A., and Pratt, S. (2002). Northern lowlands of Mars: Evidence for widespread volcanic flooding and tectonic deformation in the Hesperian Period. Journal of Geophysical Research (Planets), 107:3-1.

Head, J. W., Marchant, D. R., and Ghatan, G. J. (2004). Glacial deposits on the rim of a Hesperian-Amazonian outflow channel source trough: Mangala Valles, Mars. Geophys. Res. Lett., 31:L10701.

Hoffman, N. (2000). White Mars: A new model for Mars surface and atmosphere based in CO2. Icarus, 146:326 342.

Hourdin, F., Le Van, P., Forget, F., and Talagrand, O. (1993). Meteorological variability and the annual surface pressure cycle on Mars. J. Atmos. Sci., 50:3625-3640.

Hourdin, F., Musat, I., Bony, S., Braconnot, P., Codron, F., Dufresne, J.-L., Fairhead, L., Filiberti, M.-A., Friedlingstein, P., Grandpeix, J.-Y., Krinner, G., Levan, P., Li, Z.-X., and Lott, F. (2006). The LMDZ4 general circulation model: climate performance and sensitivity to parametrized physics with emphasis on tropical convection. Climate Dynamics, 27:787-813.

Ivanov, M. A. and Head, J. W. (2001). Chryse Planitia, Mars : Topographic configuration, outflow channel continuity and sequence, and tests for hypothesized ancient bodies of water using Mars Orbiter Laser Altimeter (MOLA) data. Journal of Geophysical Research, 106:3275-3295. 
Kerber, L., Forget, F., and Wordsworth (2015). Sulfur in the early martian atmosphere revisited : Experiments with a 3-D Global Climate Model. Icarus, 261:133-148.

Kite, E. S., Michaels, T. I., Rafkin, S., Manga, M., and Dietrich, W. E. (2011a). Localized precipitation and runoff on Mars. Journal of Geophysical Research, 116.

Kite, E. S., Rafkin, S., Michaels, T. I., Dietrich, W. E., and Manga, M. (2011b). Chaos terrain, storms, and past climate on Mars. Journal of Geophysical Research, 116.

Kite, E. S., Williams, J. P., Lucas, A., and Aharonson, O. (2014). Low palaeopressure of the martian atmosphere estimated from the size distribution of ancient craters. Nature Geoscience, 7:335-339.

Kleinhans, M. G. (2005). Flow discharge and sediment transport models for estimating a minimum timescale of hydrological activity and channel and delta formation on Mars. Journal of Geophysical Research, 110.

Knudsen, J. G. and Katz, D. L. (1958). Fluid Dynamics and Heat Transfer. McGraw-Hill, New-York.

Komatsu, G. and Baker, V. R. (1997). Paleohydrology and flood geomorphology of Ares Valles. Journal of Geophysical Research, 102.

Komatsu, G., Kargel, J. S., Baker, V. R., Strom, R. G., Ori, G. G., C., M., and L., T. K. (2000). A chaotic terrain formation hypothesis: Explosive outgas and outlow by dissociation of clathrate on Mars.

Kreslavsky, M. A. and Head, J. W. (2002). Fate of outflow channel effluents in the northern lowlands of mars: The vastitas borealis formation as a sublimation residue from frozen ponded bodies of water. Journal of Geophysical Research, 107(12).

Laskar, J., Correia, A. C. M., Gastineau, M., Joutel, F., Levrard, B., and Robutel, P. (2004). Long term evolution and chaotic diffusion of the insolation quantities of Mars. Icarus, 170:343-364.

Le Treut, H. and Li, Z. X. (1991). Sensitivity of an atmospheric general circulation model to prescribed SST changes: Feedback effects associated with the simulation of cloud optical properties. Climate Dynamics, 5:175-187. 
Leconte, J., Forget, F., Charnay, B., Wordsworth, R., and Pottier, A. (2013a). Increased insolation threshold for runaway greenhouse processes on earth-like planets. Nature, 504:268-280.

Leconte, J., Forget, F., Charnay, B., Wordsworth, R., Selsis, F., and Millour, E. (2013b). 3D climate modeling of close-in land planets: Circulation patterns, climate moist bistability and habitability. Astronomy. and Astrophysics, in press.

Manabe, S. and Wetherald, R. (1967). Thermal equilibrium of the atmosphere with a given distribution of relative humidity. J. Atmos. Sci., 24:241-259.

Mangold, N., Quantin, C., Ansan, V., Delacourt, C., and Allemand, P. (2004). Evidence for Precipitation on Mars from Dendritic Valleys in the Valles Marineris Area. Science, 305:78-81.

Mellor, G. L. and Yamada, T. (1982). Development of a turbulence closure model for geophysical fluid problems. Rev. of Geophys., 20(4):851-875.

Milton, D. J. (1974). Carbon dioxide hydrate and floods on Mars. Science, 183:654656.

Mouginot, J., Pommerol, A., Beck, P., Kofman, W., and Clifford, S. M. (2012). Dielectric map of the Martian northern hemisphere and the nature of plain filling materials. Geophysical Research Letters, 39.

Phillips, R. J., Zuber, M. T., Solomon, S. C., Golombek, M. P., Jakosky, B. M., Banerdt, W. B., Smith, D. E., Williams, R. M. E., Hynek, B. M., Aharonson, O., and Hauck, S. A. (2001). Ancient Geodynamics and Global-Scale Hydrology on Mars. Science, 291:2587-2591.

Quantin, C., Allemand, P., Mangold, N., Dromart, G., and Delacourt, C. (2005). Fluvial and lacustrine activity on layered deposits in Melas Chasma, Valles Marineris, Mars. Journal of Geophysical Research, 110.

Quantin, C., Craddock, R. A., Dubuffet, F., Lozac'h, L., and Martineau, M. (2015). Long-term evolution of the erosion rates during early mars. In European Planetary Science Congress 2015.

Ramirez, R. M. and Kasting, J. F. (2017). Could cirrus clouds have warmed early Mars? Icarus, 281:248-261. 
Rothman, L. S., Gordon, I. E., Babikov, Y., Barbe, A., Benner, D. C., Bernath, P. F., Birk, M., Bizzocchi, L., Boudon, V., Brown, L. R., Campargue, A., Chance, K., Coudert, L. H., Devi, V. M., Drouin, B. J., Fayt, A., Flaud, J.-M., Gamache, R. R., Harrison, J. J., Hartmann, J. M., Hill, C., Hodges, J. T., Jacquemart, D., Jolly, A., Lamouroux, J., Le Roi, R. J., Li, G., Long, D. A., Lyulin, O. M., Mackie, C. J., Massie, S. T., Mikhailenko, S., Mller, H. S. P., Naumenko, O. V., Nikitin, A. V., Orphal, J., Perevalov, V. I., Perrin, A., Polovtseva, E. R., Richard, C., Smith, M. A. H., Starikova, E., Sung, K., Tashkun, S. A., Tennyson, J., Toon, G. C., Tyuterev, V. G., and Wagner, G. (2013). The HITRAN2012 molecular spectroscopic database. J. Quant. Spectrosc. Radiat. Transfer, 130:4-55.

Rotto, S. L. and Tanaka, K. L. (1992). Chryse Planitia region, Mars: Channeling history, flood volume estimates, and scenarios for bodies of water in the northern plains. Lunar and Planet. Inst., Houston, Tex. Workshop on the Martian Surface and Atmosphere Through Time.

Santiago, D. L., Colaprete, A., Kreslavsky, M., Kahre, M. A., and Asphaug, E. (2012). Cloud Formation and Water Transport on Mars After Major Outflow Events. volume 43 of Lunar and Planetary Science Conference.

Smith, D. E., Zuber, M. T., and 17 coauthors (1999). The global topography of Mars and implication for surface evolution. Science, 284:1495-1503.

Smith, D. E., Zuber, M. T., Frey, H. V., Garvin, J. B., Head, J. W., Muhleman, D. O., Pettengill, G. H., Phillips, R. J., Solomon, S. C., Zwally, H. J., Banerdt, W. B., Duxbury, T. C., Golombek, M. P., Lemoine, F. G., Neumann, G. A., Rowlands, D. D., Aharonson, O., Ford, P. G., Ivanov, A. B., Johnson, C. L., McGovern, P. J., Abshire, J. B., Afzal, R. S., and Sun, X. (2001). Mars Orbiter Laser Altimeter: Experiment summary after the first year of global mapping of Mars. J. Geophys. Res., 106:23689-23722.

Spiga, A., Guerlet, S., Meurdesoif, Y., Indurain, M., Millour, E., Dubos, T., Sylvestre, M., Leconte, J., and Fouchet, T. (2015). Waves and eddies simulated by high-resolution global climate modeling of saturns troposphere and stratosphere. In European Planetary Science Congress 2015.

Tian, F., Claire, M. W., Haqq-Misra, J. D., Smith, M., Crisp, D. C., Catling, D., Zahnle, K., and Kasting, J. F. (2010). Photochemical and climate consequences 
of sulfur outgassing on early Mars. Earth and Planetary Science Letters, 295:412-418.

Toon, O. B., McKay, C. P., Ackerman, T. P., and Santhanam, K. (1989). Rapid calculation of radiative heating rates and photodissociation rates in inhomogeneous multiple scattering atmospheres. J. Geophys. Res., $94: 16,287-16,301$.

Turbet, M., Forget, F., Charnay, B., and Leconte, J. (2017). The $\mathrm{CO}_{2}$ condensation: a serious limit to the deglaciation of Earth-like planets. Earth and Planetary Science Letters. to be submitted.

Turbet, M., Leconte, J., Selsis, F., Bolmont, E., Forget, F., Ribas, I., Raymond, S. N., and Anglada-Escudé, G. (2016). The habitability of Proxima Centauri b. II. Possible climates and observability. Astronomy and Astrophysics, 596:A112.

Urata, R. A. and Toon, O. B. (2013). Simulations of the martian hydrologic cycle with a general circulation model: Implications for the ancient martian climate. Icarus, 226:229-250.

Weitz, C. M., Milliken, R. E., Grant, J. A., McEwen, A. S., Williams, R. M. E., and Bishop, J. L. (2008). Light-toned strata and inverted channels adjacent to Juventae and Ganges chasmata, Mars. Geophysical Research Letters, 35.

Wilson, L., Ghatan, G. J., Head, J. W., and Mitchell, K. L. (2004). Mars outflow channels: A reappraisal of the estimation of water flow velocities from water depths, regional slopes, and channel floor properties. Journal of Geophysical Research, 109.

Wordsworth, R., Forget, F., and Eymet, V. (2010). Infrared collision-induced and far-line absorption in dense $\mathrm{CO}_{2}$ atmospheres. Icarus, 210:992-997.

Wordsworth, R., Forget, F., Millour, E., Head, J. W., Madeleine, J.-B., and Charnay, B. (2013). Global modelling of the early martian climate under a denser $\mathrm{CO}_{2}$ atmosphere: Water cycle and ice evolution. Icarus, 222:1-19.

Wordsworth, R. D., Forget, F., Selsis, F., Millour, E., Charnay, B., and Madeleine, J.-B. (2011). Gliese 581d is the First Discovered Terrestrial-mass Exoplanet in the Habitable Zone. The Astrophysical Journal Letters, 733:L48. 